Please quote as: Dellermann, D., Lipusch, N., Ebel, P. \& Leimeister, J. M. (2021). CrowdServ - Konzept für ein hybrides Entscheidungsunterstützungssystem zur Validierung von Geschäftsmodellen. In D. Beverungen, J. H. Schumann, V. Stich \& G. Strina (ed.), Dienstleistungsinnovationen durch Digitalisierung, Vol. Band 2: Prozesse - Transformation - Wertschöpfungsnetzwerke (pp. 291-331).

Berlin/Heidelberg: Springer Gabler. ISBN: 978-3-662-63098-3 


\title{
CrowdServ - Konzept für ein hybrides Entscheidungsunterstützungssystem zur Validierung von Geschäftsmodellen
}

\author{
Dominik Dellermann, Nikolaus Lipusch, Philipp Ebel und Jan Marco \\ Leimeister
}

\section{Zusammenfassung}

Eine der kritischsten Aufgaben für Start-ups ist die Validierung ihres Geschäftsmodells. Daher versuchen Gründer, Informationen wie z. B. Feedback von anderen Akteuren zu sammeln, um die Gültigkeit ihrer Annahmen zu bewerten und Entscheidungen $\mathrm{zu}$ treffen. Bisherige Arbeiten über Entscheidungshilfen für die Validierung von Geschäftsmodellen bieten jedoch keine Lösung für den höchst unsicheren und komplexen Kontext von Start-ups in der Frühphase. Der Zweck dieser Studie ist daher die Entwicklung von Entwurfsprinzipien für ein hybrides Entscheidungsunterstützungssystem (HIDSS), das die komplementären Fähigkeiten von menschlicher und maschineller Intelligenz kombiniert. Wir verfolgen einen gestaltungsorientierten Forschungsansatz, um ein Prototyp-Artefakt und eine Reihe von Designprinzipien zu entwerfen. Unsere Studie liefert präskriptives Wissen für HIDSS und trägt zu früheren Arbeiten über Entscheidungsunterstützung für

D. Dellermann

Vencortex, Kassel, Deutschland

E-Mail: dominik.dellermann@ vencortex.com

N. Lipusch

Capgemini Applied Innovation Exchange, München, Deutschland

P. Ebel $(\square)$

Universität St.Gallen, St.Gallen, Schweiz

E-Mail: philipp.ebel@unisg.ch

J. M. Leimeister

Universität Kassel, Kassel, Deutschland

E-Mail: leimeister@uni-kassel.de

(C) Der/die Autor(en), exklusiv lizenziert durch Springer-Verlag GmbH, DE, ein Teil von 
Geschäftsmodelle, über die Anwendung der komplementären Stärken von Mensch und Maschine zur Entscheidungsfindung und über Unterstützungssysteme für extrem unsichere Entscheidungsprobleme bei.

\subsection{Einleitung}

Um die Gründung eines Start-ups voranzutreiben, wenden sich Unternehmensgründer oftmals an sogenannte Inkubatoren. Dies sind Einrichtungen oder Institutionen, die Unternehmensgründer auf verschiedene Art und Weise auf ihrem Weg in die Selbstständigkeit durch verschiedene Dienstleistungsangebote begleiten und unterstützen. Inkubatoren fördern damit gezielt Unternehmensgründungen und ihre Wachstumschancen. Durchschnittlich haben durch Inkubatoren gestützte Unternehmen eine deutlich höhere Überlebensrate, die bis zu circa $50 \%$ erreichen kann (Bøllingtoft \& Ulhøi 2005, S. 267 f.). Inkubatoren sind in der Regel eigenständige Einrichtungen oder Institutionen, die sowohl von Hochschulen als auch von Wirtschaftsverbänden betrieben werden. Inkubatoren unterstützen junge Unternehmen in den verschiedensten Bereichen. Dies fängt bei Beratung und Coaching an, geht über die Bereitstellung von Mietflächen oder ganzen Büroräumen und der Infrastrukturausstattung bis hin zu umfangreichen Service- und Dienstleistungspaketen, in deren Rahmen beispielsweise ein professioneller Businessplan erstellt werden kann oder eine juristische Beratung bei Patent- und Lizenzstrategien erfolgt. Insgesamt spielen Inkubatoren eine bedeutende Rolle innerhalb einer Volkswirtschaft und beeinflussen das Wirtschaftswachstum, schaffen Arbeitsplätze und erhöhen nachweislich die Überlebenschance von Start-ups (Aerts et al. 2007, S. 255).

Ein nachgewiesener Erfolgsfaktor bei der erfolgreichen Gründung von Unternehmen ist das frühzeitige „Testen“ der eigenen Geschäftsidee und/oder des eigenen Geschäftsmodells. Bisherige Studien legen nahe, dass Gründer, die ihre Geschäftsidee und/oder ihr Geschäftsmodell schon im frühen Stadium ihrer Existenzgründung potenziellen Kunden zur Beurteilung vorlegen, bei der Gründung ihres Unternehmens erfolgreicher sind als Gründer, die dies nicht tun (Lussier 1995, S. 8 ff.). Bedauerlicherweise fehlt es dem Dienstleistungsspektrum von Inkubatoren aber an Angeboten, die ein Testen und Bewerten von Geschäftsideen und -modellen ermöglichen. Zwar bieten einige Inkubatoren Geschäftsmodell-Ideenwettbewerbe an, die ein Bewerten und Testen in gewisser Weise ermöglichen, jedoch wird dies in der Regel nur durch eine kleine Jury nach unterschiedlichen, individuellen Bewertungskategorien vorgenommen, die nicht unbedingt die Meinung potenzieller Kunden widerspiegeln. Ein solches kundenbasiertes Feedback ist wichtig, da Geschäftsmodell und Geschäftsidee nicht nur am Kunden ausgerichtet werden sollten, sondern Kunden auch über Lösungs- und Bedürfnisinformationen verfügen, welche zur Verbesserung von sowohl Geschäftsidee als auch Geschäftsmodell herangezogen werden können. Der Grund dafür, dass Inkubatoren Dienstleistungen, die ein kundenbasiertes Feedback der Geschäftsideen oder -modelle 
ermöglichen, bislang nicht anbieten, liegt sicherlich daran, dass es schlichtweg an den dafür notwendigen Infrastrukturen und Möglichkeiten fehlt.

Das Ziel dieses Forschungsvorhabens war es daher, ein Entscheidungsunterstützungssystem (Decision Support System, DSS) zu entwickeln, das die iterative Validierung eines Geschäftsmodells durch die Kombination von sozialer Interaktion mit relevanten Stakeholdern (z. B. Partnern, Investoren, Mentoren und Kunden) und einer formalen Analyse für den extrem unsicheren Kontext der Geschäftsmodellentwicklung in der Frühphase von Unternehmensgründungen ermöglicht. Eine solche Kombination hat sich als äußerst wertvoll für Entscheidungen in diesem Umfeld erwiesen (z. B. Huang \& Pearce 2015, S. 658 f.). Insbesondere schlagen wir ein Hybrid Intelligence DSS (HIDSS) vor, das die Stärke sowohl der maschinellen Intelligenz zur Handhabung großer Informationsmengen als auch der kollektiven Intelligenz kombiniert, die die Intuition und das kreative Potenzial des Einzelnen nutzt und gleichzeitig systematische Fehler durch statistische Mittelung reduziert. Wir verfolgen einen gestaltungsorientierten Ansatz (Hevner 2007, S. 87 f.; Peffers et al. 2007, S. 49), wobei wir sowohl Wissen aus der bisherigen Forschung, das sich in verschiedenen Kontexten unsicherer Entscheidungsfindung als wertvoll erwiesen hat, als auch praktische Erkenntnisse nutzen, um Designprinzipien für ein HIDSS zu entwickeln.

Unser Beitrag ist dreifach. Erstens liefert unsere Forschung präskriptives Wissen, das als Blaupause für die Entwicklung ähnlicher DSS für die Validierung von Geschäftsmodellen im Kontext von Start-ups in der Frühphase dienen kann (Gregor \& Hevner 2013, S. 343). Tatsächlich liefert unsere Forschung präskriptives Wissen sowohl über die Designprinzipien (d. h. Form und Funktion) als auch über die Implementierungsprinzipien (d. h. die von uns vorgeschlagene Implementierung). Zweitens leisten wir einen Beitrag zur Forschung über die Entscheidungsunterstützung für die Validierung von Geschäftsmodellen, indem wir die formale Analyse von Daten durch iterative soziale Interaktion mit Interessengruppen ergänzen. Drittens schlagen wir einen neuartigen Ansatz zur Unterstützung der menschlichen Entscheidungsfindung durch die Kombination von maschineller und kollektiver Intelligenz vor und tragen so zur neueren Forschung über Ensemble-Methoden bei (z. B. Nagar \& Malone 2011, S. 12).

\subsection{Bisherige Forschungsarbeiten}

\subsubsection{Geschäftsmodelle und deren Validierung}

Um das Problem zu formulieren, haben wir die aktuelle Literatur über die Validierung von Geschäftsmodellen analysiert. Das Konzept des Geschäftsmodells hat in den letzten Jahren sowohl in der Wissenschaft als auch in der Praxis große Aufmerksamkeit erlangt (Veit et al. 2014, S. 45 f.). Im Allgemeinen beschreibt es die Logik eines Unternehmens, Wert zu schaffen und zu erfassen (Al-Debei \& Avison 2010, S. 360 f.; Zott et al. 2011, S. 1022 f.). Das Konzept des Geschäftsmodells bietet einen umfassenden Ansatz zur 
Beschreibung der Wertschöpfung für alle involvierten Stakeholder und der Verteilung der Aktivitäten unter ihnen (Bharadwaj et al. 2013, S. 477 f.; Demil et al. 2015, S. 227).

Im Kontext von Neugründungen werden Geschäftsmodelle besonders relevant, da die Unternehmer ihre Vorstellungen davon, wie die Marktbedürfnisse zukünftiger Kunden bedient werden sollen, genauer definieren. Ein Geschäftsmodell spiegelt die Annahmen eines Unternehmers wider und kann daher als eine Reihe von Hypothesen darüber betrachtet werden, was die Kunden wollen und wie ein Unternehmen diese Bedürfnisse am besten erfüllen und dafür bezahlt werden kann (Teece 2010, S. 191). Dabei treffen Unternehmer mehrere Entscheidungen über die Gestaltung eines Geschäftsmodells, wie z. B. über die Art und Weise, wie ein Ertragsmodell, ein Wertversprechen und Kundenkanäle aufgebaut werden sollen. So kann ein Geschäftsmodell als Rahmen für die Konstruktion von Start-ups und für die Durchführung einer vorausschauenden Waswäre-wenn-Szenarioanalyse verwendet werden, um die Durchführbarkeit des aktuellen Weges eines Unternehmers zu bestimmen (Morris et al. 2005, S. 733). Allerdings sind solche Szenarioanalysen bezüglich der Annahmen eines Unternehmers darüber, was machbar und durchführbar sein könnte, im Hinblick auf das Ergebnis meist kurzsichtig, weil Unternehmer unter einem hohen Maß an Unsicherheit agieren (Alvarez \& Barney 2007, S. 18). Folglich müssen Unternehmer einen Prozess der Sensibilisierung beginnen, um Informationen zur Validierung und Verfeinerung ihrer anfänglichen Überzeugungen und zur Orientierung für zukünftige Entscheidungen zu sammeln. Während dieses Prozesses verfeinern die Unternehmer das Geschäftsmodell durch iterative Experimente und das Lernen aus erfolgreichen und gescheiterten Handlungen. Diese Designentscheidungen bestimmen, wie ein Geschäftsmodell entlang mehrerer Dimensionen konfiguriert wird (Alvarez et al. 2013; Blank 2013). Wenn die Annahmen der Unternehmer im Widerspruch zur Reaktion des Marktes stehen, kann dies zu einer Ablehnung falscher Hypothesen führen und eine Neubewertung des Geschäftsmodells erfordern, um die Marktwahrnehmung erneut zu testen. So entwickelt sich das Geschäftsmodell in Richtung der Bedürfnisse des Marktes und verändert die Annahmen der Unternehmer (Ojala 2016, S. 454). Der Erfolg von Start-ups hängt also stark von der Fähigkeit der Unternehmer ab, ihre Geschäftsmodelle zu entwickeln und durch geeignete Entscheidungen kontinuierlich an die Reaktionen des Umfelds anzupassen (Spiegel et al. 2016, S. 435 ff.).

\subsubsection{Entscheidungshilfe für die Validierung von Geschäftsmodellen}

Bei der Entscheidungsfindung für den Entwurf von Geschäftsmodellen (d. h. wie ein Geschäftsmodell konstruiert werden sollte) werden innerhalb der existierenden Literatur verschiedene Maßnahmen zur Entscheidungsunterstützung diskutiert.

Zum einen konzentriert sich die bisherige Forschung zur Entscheidungsunterstützung und Validierung im Kontext der Geschäftsmodellanalyse hauptsächlich auf analytische 
Methoden wie Modellierung und Simulation (z. B. Gordijn et al. 2001, S. 13 ff.; Haaker et al. 2017, S. 17 ff.; Daas et al. 2013, S. 253 f.; Euchner \& Ganguly 2014, S. 37). Geschäftsmodellsimulationen bieten einen zeit- und kosteneffizienten Weg, um Entscheidungsträgern zu helfen, die Konsequenzen von Geschäftsmodellanpassungen zu verstehen, ohne dass umfangreiche organisatorische Veränderungen erforderlich sind (Osterwalder et al. 2005, S. 26 ff.). In diesem Sinne wendet die bisherige Forschung quantitative Szenarioanalysen an, um die Tragfähigkeit von Designentscheidungen im Kontext von Geschäftsmodellinnovationen für Plattformen (Zoric 2011, S. 48 ff.) und mobiles Fernsehen (Pagani 2009, S. 384 ff.) sowie Szenarioplanungsmethoden für IPfähige TV-Geschäftsmodelle vorherzusagen (Bouwman et al. 2008, S. 31 ff.). Eine weitere Möglichkeit, eine Anleitung für die Gestaltung eines Geschäftsmodells zu geben, konzentriert sich auf die stochastische Analyse von Finanzmodellen, um die wichtigsten Triebkräfte für die finanzielle Leistungsfähigkeit etablierter Unternehmen zu identifizieren (Euchner \& Ganguly 2014, S. 34 ff.). Ein dritter populärer Ansatz bewertet die Wahl des Geschäftsmodell-Designs im Hinblick auf ein potenzielles Szenario von Veränderungen in Stresstestfällen (Haaker et al. 2017, S. 17 ff.).

Obwohl der Großteil dieser Forschung die Bedeutung der Konsistenz kausaler Geschäftsmodellstrukturen und der komplexen Wechselbeziehungen der Komponenten berücksichtigt, berücksichtigen die bestehenden Methoden nicht, wie sich die Auswirkungen von Änderungen in einem Geschäftsmodell im Laufe der Zeit dynamisch entfalten und den iterativen Prozess der Entwicklung von Geschäftsmodellen insbesondere für neue Unternehmen beeinflussen (Cavalcante et al. 2011, S. 1327 f.; Demil \& Lecocq 2010, S. 227 ff.). Die meisten dieser Ansätze sind eher statisch und daher nur wenig in der Lage, die Dynamik zu erfassen, die den komplexen Wechselwirkungen von Entscheidungen zur Geschäftsmodellgestaltung in der Praxis zugrunde liegt. Solchen analytischen Methoden zur Unterstützung von Entscheidungen im Rahmen der Geschäftsmodell-Validierung fehlt die Fähigkeit, komplexe Muster von Komponenten zu identifizieren, die zum Erfolg führen.

Während diese Methoden für etablierte Unternehmen wertvoll sind, sind sie für Start-ups in der Frühphase kaum anwendbar. Die Vorhersage des Erfolgs von Geschäftsmodellen in der Frühphase von Unternehmen ist äußerst komplex und unsicher. Dies liegt daran, dass weder mögliche Ergebnisse noch die Wahrscheinlichkeit, diese Ergebnisse zu erreichen, bekannt sind, d. h. es geht um Situationen mit nicht erklärbaren Risiken (Alvarez \& Barney 2007, S. 301 ff.). Es sind nur wenige Daten verfügbar, und eine Quantifizierung der Wahrscheinlichkeit bestimmter Ereignisse bleibt unmöglich. In solchen Kontexten ist eine formale Analyse eine notwendige, aber nicht ausreichende Bedingung, um zu beurteilen, ob ein bestimmtes Geschäftsmodell in der Zukunft tragfähig sein könnte (Huang \& Pearce 2015, S. 657). In solchen Situationen verfolgen die Unternehmer zwei Strategien. Erstens suchen und sammeln sie verfügbare Informationen, die sie verarbeiten können, um die analytische Entscheidungsfindung zu leiten (Shepherd et al. 2015, S. 32). Zweitens verlassen sich Unternehmer auf ihre Erfahrung und ihr Bauchgefühl, um intuitiv fundierte Entscheidungen zu treffen. Eine 
solche Intuition hat sich als wertvolle Strategie für die Entscheidungsfindung unter Unsicherheit erwiesen (Huang \& Pearce 2015, S. 658).

Während sich erfolgreiche Unternehmer bei der Validierung ihrer Geschäftsmodelle auf ihr Bauchgefühl und ihre intuitive Entscheidungsfindung verlassen, argumentieren wir, dass die Bewertung, Verarbeitung und Interpretation zusätzlicher Informationen, die die Unsicherheit verringern und die Entscheidungsfindung leiten können, Unterstützung benötigt (March 1978, S. 587 ff.; Simon 1955, S. 99 ff.). Dies liegt daran, dass Unternehmensgründer in ihrer Fähigkeit, auf Informationen umfassend zuzugreifen und sie zu verarbeiten, eingeschränkt und daher nicht in der Lage sind, ihre Entscheidungen zu optimieren. Darüber hinaus unterliegt die Interpretation der abgerufenen und verarbeiteten Informationen Verzerrungen und Heuristiken, was häufig zu schlechten Entscheidungen führt. Da Unternehmensgründer dadurch in ihrer Rationalität eingeschränkt sind, hat sich das Start-up-Mentoring als Strategie zur Unterstützung von Unternehmern bei den erforderlichen Entscheidungen herausgebildet. Mentoren (d. h. erfahrene Berater, Experten oder erfolgreiche Unternehmer) versuchen, das Start-upTeam in der Frühphase dabei zu unterstützen, die Problemlösung sauber zu definieren, indem sie individuelle Unterstützungsinitiativen (z. B. Workshops) durchführen und den Unternehmern Methoden anbieten, um ihre Idee zu einem neuartigen Unternehmen zu entwickeln (Cohen \& Hochberg 2014, S. 4 f.). Eine solche Interaktion mit relevanten Interessengruppen wird häufig von Dienstleistern wie Inkubatoren und in jüngerer Zeit von Acceleratoren angeboten (z. B. Cohen \& Hochberg 2014, S. 1 ff.; Pauwels et al. 2016, S. 13 ff.). Allerdings bieten weder die Wissenschaft noch die Managementpraxis IT-gestützte Lösungen an, um eine solche Beratung iterativ zu ermöglichen. Dies ist bedauerlich, da IT-basierte Lösungen das Potenzial haben, skalierbare und kosteneffiziente Lösungen zu bieten, indem sie die Weisheit mehrerer und unterschiedlicher Mentoren nutzen, den Validierungs- und Anpassungsprozess iterativ gestalten, die Übertragung der Erfahrungen vieler Unternehmer auf einen einzigen Unternehmer ermöglichen und dadurch die Lernrate des einzelnen Unternehmers erhöhen.

\subsection{Methodisches Vorgehen - Gestaltungsorientierte Forschung}

Wie in den vorgegangenen Abschnitten skizziert, werden neue Lösungen benötigt, um die Grenzen der einzelnen Entscheidungsträger zu überwinden, die sich aus ihrer begrenzten Rationalität und dem Mangel an skalierbaren Lösungen für die Beratung in Gründerzentren und Acceleratoren ergeben.

Um Unternehmern IT-gestützte Formen der Beratung zu bieten, haben wir ein gestaltungsorientiertes Forschungsprojekt (DSR) durchgeführt (Peffers et al. 2007 S. 46 ff.; Gregor \& Hevner 2013, S. 4), um ein neues und innovatives Artefakt zu entwerfen, das zur Lösung eines realen Problems beiträgt, indem es ein qualitativ hochwertiges und skalierbares Werkzeug als Entscheidungshilfe bereitstellt. Für unsere 
Forschung folgten wir dem iterativen DSR-Prozess von Peffers et al. (2007, S. 54), der aus sechs Phasen besteht: 1) Problemidentifikation und -motivation, 2) Identifizierung der Ziele der zu entwickelnden Lösung, 3) Design und Entwicklung der Lösung, 4) Demonstration der Lösung, 5) Evaluation und 6) Kommunikation der Lösung. Wir verwendeten eine mehrstufige Evaluierung mit Fachexperten und potenziellen Nutzern, um die Validität unserer Ergebnisse sicherzustellen (Venable et al. 2016, S. 79).

Unsere Forschung beginnt mit Phase 1, d. h. der Formulierung des in der Praxis wahrgenommenen Problems. Um sowohl Relevanz als auch Rigorosität zu gewährleisten, verwenden wir für unser Forschungsprojekt Inputs aus dem praktischen Problembereich (Relevanz) und dem vorhandenen Wissensbestand (Rigorosität) (Hevner 2007, S. 89 f.). Abstraktes theoretisches Wissen hat hierbei eine doppelte Funktion. Erstens befasst es sich mit den Vorschlägen für eine mögliche Lösung. Zweitens dient das abstrakte Lernen aus unserem Entwurf als präskriptives Wissen, um ein ähnliches Artefakt in der Zukunft zu entwickeln (Gregor \& Hevner 2013, S. 343). Deshalb haben wir eine Literaturrecherche zur Entscheidungsunterstützung im Kontext der Validierung von Geschäftsmodellen durchgeführt. Um die Relevanz des Problems zu verfeinern und zu validieren, führten wir eine explorative Studie innerhalb der Problemdomäne mithilfe qualitativer Interviews durch. Wir sammelten Daten über den Prozess der Geschäftsmodell-Validierung innerhalb von Acceleratoren und Inkubatoren und führten eine Reihe von Experteninterviews mit Führungskräften in Geschäftsinkubatoren und -acceleratoren $(n=27)$, Unternehmern $(n=32)$ und Mentoren $(n=16)$ durch. Die Aussagen der Befragten wurden von zwei der Forscher kodiert und analysiert, um gemeinsame Themen zu identifizieren. Unser Kodierungsverfahren war strukturiert und orientierte sich an den aus der Literatur abgeleiteten Einschränkungen (Strauss \& Corbin 1990, S. 220 ff.). Dieser Ansatz erlaubte es uns, vor dem Entwerfen des Artefakts die Praxisrelevanz der Forschungslücke zu verifizieren.

In einem zweiten Schritt analysierten wir die bisherige Forschung zur Entscheidungsunterstützung, um einen Wissensfundus zu identifizieren, der Vorschläge für eine wissenschaftlich fundierte Version der Entwurfsprinzipien liefert. Zur Bewertung unseres Designs verwendeten wir eine Kombination aus explorativen und bestätigenden Fokusgruppen. Ursprünglich aus dem Bereich der Psychologie stammend, gewinnt die Fokusgruppe als Technik zur Wissenserhebung auch im Bereich des Software-Engineering zunehmend an Popularität (Massey \& Wallace 1991, S. 193 ff.; Nielsen 1997, S. 94 f.). Wir verwendeten explorative Fokusgruppen, um Feedback für Designänderungen und die Verfeinerung des Artefakts zu sammeln. Dies wurde als formatives Bewertungsverfahren zur iterativen Verbesserung des Designs verwendet. Darüber hinaus wurde eine konfirmatorische Fokusgruppe eingesetzt, um den Nutzen unseres Artefaktentwurfs im Anwendungsbereich zu demonstrieren. Die erste Version der vorläufigen Designprinzipien wurde in acht Fokusgruppen-Workshops (6-8 Teilnehmer; durchschnittliche Dauer 60 min) mit Mentoren, Führungskräften und Softwareentwicklern demonstriert, validiert und verfeinert. Die Entwurfsprinzipien wurden visualisiert, erklärt und diskutiert, um die Vollständigkeit, innere Konsistenz und Anwendbarkeit unseres 
Ex-Ante-Entwurfs formativ zu bewerten (Venable et al. 2016, S. 79). In den nächsten Schritten instanziierten wir die vorläufigen Designprinzipien von Form und Funktion in ein IT-Artefakt. Anschließend führten wir eine summative Ex-Post-Evaluierung des Entwurfs durch eine qualitative Bewertung in einem naturalistischen Umfeld mit potenziellen Nutzern durch. Dazu veranstalteten wir acht Fokusgruppen-Workshops mit Mentoren, Führungskräften von Inkubatoren und Unternehmensbeschleunigern sowie Unternehmern (2-4 Teilnehmer; durchschnittliche Dauer 60 min). Im Rahmen dieser Fokusgruppen wurde das instanziierte Artefakt den Teilnehmern erklärt und durch ein Walkthrough demonstriert. Die Teilnehmer hatten im Anschluss daran die Möglichkeit, das Artefakt zu benutzen, und wurden dann gebeten, seine Effektivität, Effizienz und Übereinstimmung mit Phänomenen der realen Welt zu bewerten, was zu einer endgültigen Version der abgeleiteten Designprinzipien führte (Venable et al. 2016, S. 79).

Abb. 8.1 fasst die sechs verschiedenen Phasen und ihre Aktivitäten zusammen, wie sie im Laufe dieses DSR-Projekts durchgeführt wurden.

\subsection{Problemverifizierung (Phase 1): Die Validierung von Geschäftsmodellen in der Frühphase bestehender Mentoringprogramme}

Um eine möglichst ganzheitliche Perspektive auf das Problem zu ermöglichen und die praktische Relevanz der identifizierten Lücke zu gewährleisten, führten wir insgesamt 75 Sondierungsinterviews mit Gründern und Mitgliedern von Inkubatoren durch. Die Interviews wurden von der zentralen Frage geleitet, wie Inkubatoren als Dienstleister typischerweise die Gestaltungsentscheidungen der Geschäftsmodelle von Unternehmern unterstützen und welche Grenzen diese Ansätze haben. Daher wurden die Interviews
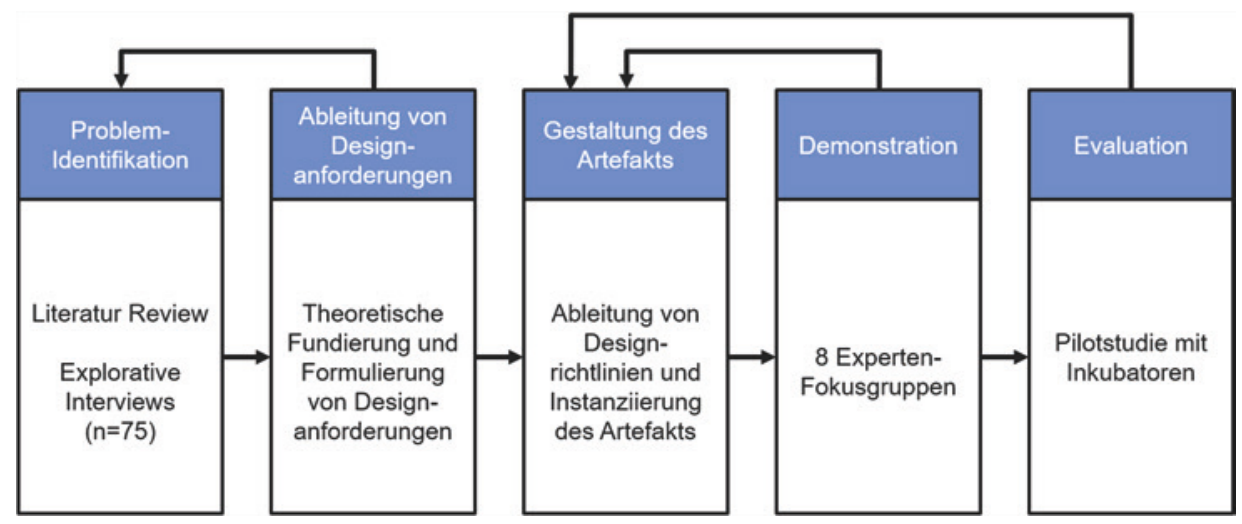

Abb. 8.1 Methodisches Vorgehen und Aktivitäten innerhalb des Forschungsvorhabens. (In Anlehnung an Peffers et al. 2007) 
zum Verständnis der Problemdomäne und zum Sammeln tiefer Einblicke in die reale Welt genutzt. Durch die Analyse dieser Interviews gewannen wir ein tieferes Verständnis des Prozesses der Validierung von Geschäftsmodellen für Start-ups.

Zusammenfassend stellte sich heraus, dass Unternehmer mit drei Arten von Problemen konfrontiert sind, wenn sie versuchen, die Qualität ihrer Geschäftsmodelle zu erhöhen. Die erste Art von Problemen betrifft die begrenzte Rationalität der Menschen, die sie an einer umfassenden Suche nach den erforderlichen Informationen hindert und zu verzerrten Entscheidungen führt. Ein zweiter Typ betrifft die Beschränkungen der gegenwärtigen Formen der Entscheidungshilfe für Unternehmer (z. B. Mentoring in Gründerzentren), die die Mentoren daran hindern, optimale Lösungen $\mathrm{zu}$ finden. $\mathrm{Zu}$ diesen Einschränkungen gehören ein begrenzter Wissensbereich, insbesondere in Bezug auf neue Technologien, ein Mangel an kognitiver Flexibilität und die Subjektivität der Bewertungen. Darüber hinaus machen es die Ressourcenbeschränkungen institutioneller Mentoren-Organisationen im Allgemeinen fast unmöglich, die perfekte Entscheidungshilfe für jeden Fall eines Geschäftsmodells zu finden, und behindern die iterative Entwicklung. Die dritte Art von Problemen befasst sich mit den Beschränkungen der bestehenden IT-basierten Werkzeuge, wie im vorigen Abschnitt diskutiert.

Tab. 8.1 fasst die aggregierten Ergebnisse unserer in Abschn. 8.2 diskutierten Literaturanalyse zusammen und ergänzt sie um die Ergebnisse der Interviews. Auf dieser Grundlage ziehen wir Schlussfolgerungen für die daraus resultierenden Ziele. Zusammenfassend liefern die Interviews einen detaillierten Überblick über die Probleme, mit denen Unternehmer konfrontiert sind, wenn sie versuchen, die Qualität ihres Geschäftsmodells zu beurteilen. Allerdings konnten wir keine Erkenntnisse darüber gewinnen, wie dieser Entscheidungsprozess mit Hilfe eines IT-Tools unterstützt werden kann. Wir untersuchten daher bestehende Designtheorien, die zur Lösung ähnlicher Probleme verwendet wurden (Gregor \& Hevner 2013, S. 343 f.).

\subsection{Ableitung der Ziele der Lösung (Phase 2)}

Wie dargelegt, müssen Unternehmer für eine erfolgreiche Ausgestaltung ihres Geschäftsmodells die Qualität ihrer Entscheidungen verbessern (Sosna et al. 2010, S. 383 f.). Dies liegt auf der Hand, da die geeigneten Entscheidungen zu einem bestimmten Zeitpunkt zu treffen die vielleicht schwierigste Aufgabe für Unternehmer ist (Alvarez et al. 2013, S. 301 ff.). Entscheidungen bezüglich bestimmter Komponenten des Geschäftsmodells können den Unternehmer dabei unterstützen oder daran hindern, wichtige Meilensteine zu erreichen, wie z. B. eine tragfähige Marktposition oder eine externe Finanzierung (Morris et al. 2005, S. 727). Um die Entscheidungsträger bei der Ausführung ihrer Aufgaben zu unterstützen, müssen die Entscheidungshilfen gewisse Gestaltungsmerkmale aufweisen, die sich in unterschiedlichen Dimensionen kategorisieren lassen (Morana et al. 2017, S. 36): 
Tab. 8.1 Aggregierte Ergebnisse der Literaturanalyse

\begin{tabular}{|c|c|c|c|c|}
\hline Kategorie & Problem & Beispiel & $\begin{array}{l}\text { Identifizierte } \\
\text { Literatur }\end{array}$ & $\begin{array}{l}\text { Abgeleitete } \\
\text { Insights }\end{array}$ \\
\hline \multirow[t]{2}{*}{$\begin{array}{l}\text { Defizite aufseiten } \\
\text { des Gründers }\end{array}$} & $\begin{array}{l}\text { Limitierte } \\
\text { Expertise }\end{array}$ & $\begin{array}{r}\text { - Fehlende } \\
\text { Expertise }\end{array}$ & $\begin{array}{l}\text { Larrick und Feiler } \\
\text { (2015), Shepherd } \\
\text { et al. (2015), } \\
\text { Alvarez et al. } \\
\text { (2013) und Blank } \\
(2013)\end{array}$ & \multirow[t]{2}{*}{$\begin{array}{l}\text { Bedarf an Ent- } \\
\text { scheidungshilfen }\end{array}$} \\
\hline & $\begin{array}{l}\text { Begrenzte } \\
\text { Rationalität }\end{array}$ & $\begin{array}{l}\text { - Selbstüber- } \\
\text { schätzung } \\
\text { - Illusion von } \\
\text { Kontrolle } \\
\text { - Verfügbarkeit }\end{array}$ & $\begin{array}{l}\text { March (1978), } \\
\text { Simon (1955), } \\
\text { Tversky und } \\
\text { Kahneman (1974) } \\
\text { und Zhang und } \\
\text { Cueto (2017) }\end{array}$ & \\
\hline \multirow[t]{4}{*}{$\begin{array}{l}\text { Defizite } \\
\text { existierender Ent- } \\
\text { scheidungshilfen }\end{array}$} & $\begin{array}{l}\text { Limitierte } \\
\text { Expertise }\end{array}$ & $\begin{array}{r}\text { - Fehlende } \\
\text { Expertise }\end{array}$ & $\begin{array}{l}\text { Larrick und Feiler } \\
(2015), \text { Shepherd } \\
\text { et al. (2015) und } \\
\text { Alvarez und } \\
\text { Barney (2007) }\end{array}$ & \multirow[t]{4}{*}{$\begin{array}{l}\text { Bedarf an } \\
\text { IT-basierten } \\
\text { Lösungen und } \\
\text { Aggregation von } \\
\text { Einzelurteilen }\end{array}$} \\
\hline & $\begin{array}{l}\text { Begrenzte } \\
\text { Rationalität }\end{array}$ & $\begin{array}{l}\text { - Nutzung } \\
\text { fehlerhafter } \\
\text { Heuristiken } \\
\text { - Similarity Bias } \\
\text { - Selbstüber- } \\
\text { schätzung }\end{array}$ & $\begin{array}{l}\text { March (1978), } \\
\text { Simon (1955), } \\
\text { Tversky und } \\
\text { Kahneman (1974) } \\
\text { und Zhang und } \\
\text { Cueto (2017) }\end{array}$ & \\
\hline & $\begin{array}{l}\text { Limitierte } \\
\text { kognitive } \\
\text { Flexibilität }\end{array}$ & $\begin{array}{l}\text { - Funktionelle } \\
\text { Fixierung }\end{array}$ & $\begin{array}{l}\text { Larrick und Feiler } \\
\text { (2015) und Zhang } \\
\text { und Cueto (2017) }\end{array}$ & \\
\hline & $\begin{array}{l}\text { Ressourcen-Eng- } \\
\text { pässe }\end{array}$ & $\begin{array}{l}\text { - Mangelnder } \\
\text { Zugang zu } \\
\text { geeigneten } \\
\text { Experten } \\
\text { - Zeitmangel }\end{array}$ & $\begin{array}{l}\text { Cohen und Hoch- } \\
\text { berg (2014) und } \\
\text { Pauwels et al. } \\
\text { (2016) }\end{array}$ & \\
\hline
\end{tabular}


Tab. 8.1 (Fortsetzung)

\begin{tabular}{|c|c|c|c|c|}
\hline Kategorie & Problem & Beispiel & $\begin{array}{l}\text { Identifizierte } \\
\text { Literatur }\end{array}$ & $\begin{array}{l}\text { Abgeleitete } \\
\text { Insights }\end{array}$ \\
\hline \multirow[t]{3}{*}{$\begin{array}{l}\text { Defizite } \\
\text { existierender } \\
\text { IT-Tools }\end{array}$} & $\begin{array}{l}\text { Fokus auf } \\
\text { Finanzdaten und } \\
\text { Simulationen }\end{array}$ & $\begin{array}{l}\text { - Finanzielle } \\
\text { Vorhersagen für } \\
\text { Start-ups nicht } \\
\text { verfügbar }\end{array}$ & $\begin{array}{l}\text { Gordijn et al. } \\
\text { (2001), Haaker } \\
\text { et al. (2017), Daas } \\
\text { et al. (2013), } \\
\text { Euchner und } \\
\text { Ganguly (2014) } \\
\text { und Gordijn } \\
\& \text { Akkermans } \\
(2001)\end{array}$ & \multirow[t]{3}{*}{$\begin{array}{l}\text { Notwendig- } \\
\text { keit eines DSS } \\
\text { zur Erfassung } \\
\text { extremer } \\
\text { Unsicherheiten } \\
\text { und komplexer } \\
\text { Interaktionen }\end{array}$} \\
\hline & $\begin{array}{l}\text { Limitierte } \\
\text { Integration } \\
\text { qualitativer } \\
\text { Kriterien }\end{array}$ & $\begin{array}{l}\text { • Keine Ver- } \\
\text { wendung von } \\
\text { „weichen“ } \\
\text { Faktoren wie } \\
\text { Teamzusammen- } \\
\text { setzung und } \\
\text { Innovationsgrad }\end{array}$ & $\begin{array}{l}\text { Euchner und } \\
\text { Ganguly }(2014) \\
\text { und Dellermann } \\
\text { et al. }(2017 a, b)\end{array}$ & \\
\hline & $\begin{array}{l}\text { Limitierte Fähig- } \\
\text { keit, komplexe } \\
\text { Sachverhalte } \\
\text { darzustellen }\end{array}$ & $\begin{array}{l}\text { - Keine Abbildung } \\
\text { der Interaktionen } \\
\text { zwischen } \\
\text { einzelnen } \\
\text { Bestandteilen } \\
\text { des Geschäfts- } \\
\text { modells }\end{array}$ & $\begin{array}{l}\text { Möllers et al. } \\
\text { (2017) }\end{array}$ & \\
\hline
\end{tabular}

1. Die Beratung hat das Ziel, den Benutzer bei der Wahl der durchzuführenden Tätigkeit oder der zu treffenden Entscheidungen zu unterstützen.

2. Der Aufrufstil definiert, wie der Benutzer auf die Entscheidungshilfe zugreifen kann, z. B. automatisch, durch den Benutzer aufgerufen oder intelligent.

3. Die Anleitung kann zu verschiedenen Zeitpunkten erfolgen, z. B. während, vor oder nach der eigentlichen Aktivität. Der Zeitpunkt für den Kontext der Wahl des Geschäftsmodell-Designs sollte nicht spezifisch sein, der Zugang sollte zu verschiedenen Zeitpunkten während des Prozesses möglich sein.

4. Entscheidungshilfen können für Neulinge und Experten bereitgestellt werden. Diese Dimension von Designmerkmalen ist für unseren Kontext nicht relevant, da Unternehmer in ihrem Fachwissen von Neulingen bis hin zu Experten und Serienunternehmern variieren können.

5. Die Frage der Vertrauensbildung wird durch Entscheidungshilfen zur Gestaltung von Geschäftsmodellen nicht explizit abgedeckt. Da dies auch im traditionellen Offline-Mentoring der Fall ist, postulieren wir, dass Vertrauensfragen - obwohl sie für die zukünftige Forschung sehr relevant sind - außerhalb des Rahmens dieses Artikels liegen. 
6. Schließlich wird die inhaltliche Dimension der Entscheidungshilfe als Zweck der Beratung definiert. Da dies in hohem Maße mit der Absicht der Entscheidungshilfe zusammenhängt, haben wir dieses Gestaltungsmerkmal nicht explizit als Voraussetzung für unsere Lösung hervorgehoben.

\subsubsection{Ableitung von Zielen auf Basis existierender Literatur zur Entscheidungsunterstützung}

Für den Umfang dieser Untersuchung haben wir uns auf vier Designziele konzentriert, die für die Entscheidungsunterstützung von Geschäftsmodellen besonders relevant sind: die Form, den Modus, die Absicht und das Format der Entscheidungshilfe.

Die Entscheidungshilfe kann in zwei Formen unterteilt werden: informative Leitlinien, die dem Nutzer zusätzliche Informationen liefern, und suggestive Leitlinien, die eine Anleitung für eine geeignete Vorgehensweise bieten. Informative Beratung kann als Expertenrat verstanden werden, der deklaratives oder definitorisches Wissen vermittelt und damit dem Benutzer hilft, das Entscheidungsmodell besser zu verstehen (Limayem \& DeSanctis 2000, S. 386 ff.). Suggestive Guidance gibt spezifische Empfehlungen, wie der Benutzer handeln sollte (Arnold et al. 2004, S. 1 ff.). Diese Form der Anleitung kann die Entscheidungsqualität verbessern und den Ressourcenbedarf für die Entscheidungsfindung verringern (Montazemi et al. 1996, S. 181 ff.).

Der Modus beschreibt, wie die Anleitung funktioniert und für den Benutzer generiert wird. Sie kann vom Designer vordefiniert werden und somit a priori statisch in ein System implementiert werden oder dynamisch vom Benutzer gelernt werden und Entscheidungshilfen bei Bedarf und partizipativ in Abhängigkeit von den Eingaben des Benutzers erzeugen (Silver 1991, S. 105 ff.). Eine dynamische Anleitung ist besonders nützlich, um die Entscheidungsqualität, das Lernen der Benutzer und die Entscheidungsleistung $\mathrm{zu}$ verbessern, während eine partizipative Anleitung auch die Leistung der Benutzer bei der Lösung komplexer Aufgaben erhöht (Parikh et al. 2001, S. 303 ff.).

Die Absicht beschreibt, warum den Nutzern Beratung angeboten wird, zum Beispiel zur Klärung, zur Erweiterung von Wissen, zum Lernen oder um Empfehlungen zu geben (Arnold et al. 2004, S. 1 ff.; Gönül et al. 2006, S. 1481 ff.). Eine Entscheidungshilfe kann also mit der Absicht bereitgestellt werden, spezifische Handlungsempfehlungen oder Expertenrat zu geben, um den Benutzern bei der Lösung von Problemen und der Entscheidungsfindung zu helfen.

Das Format bezieht sich auf die Art und Weise, wie die Anleitung dem Benutzer vermittelt wird. Entscheidungshilfen können beispielsweise als reiner Text oder als Multimedia-Präsentation (Bilder, Animationen, Audio) formatiert werden, um sie für den Benutzer leichter zugänglich zu machen. Das Format der Anleitung sollte in Abhängigkeit von den Merkmalen der zugrunde liegenden Aufgabe gewählt werden (Gregor \& Benbasat 1999, S. 503). 


\subsubsection{Formulierung von Designanforderungen}

Für ein System, das Gründer bei der Entscheidungsfindung im Rahmen der Geschäftsmodell-Validierung unterstützen soll, ist im Allgemeinen eine bestimmte Kombination von kontextspezifischen Anleitungen erforderlich (Silver 1991, S. 105 ff.). Für die Zwecke unserer Forschung haben wir unsere Designanforderungen (Design Requirements, DR) entlang der vier Dimensionen strukturiert, die als geeignet für die Klasse des Entscheidungsproblems identifiziert wurden (Form, Modus, Absicht, Format).

Erstens muss die Form der gegebenen Anleitung Informationen über die Erfolgswahrscheinlichkeit der aktuellen Version des Geschäftsmodells enthalten. Sie muss eine Prognose über die Wahrscheinlichkeit des zukünftigen Erfolgs, wie z. B. den Erhalt von Finanzmitteln, das Überleben, das Wachstum usw., ermöglichen. D. h., es muss eine informative Anleitung sein. Daher formulieren wir folgende Gestaltungsanforderung:

- DR1: Die Validierung des Geschäftsmodells sollte durch ein DSS unterstützt werden, das eine informative Anleitung liefert, um den Wert des Geschäftsmodells zu signalisieren.

Zweitens sollte das DSS die Anpassung des Geschäftsmodells durch den Unternehmer anleiten, indem es konkrete Ratschläge gibt, wie die Elemente des Geschäftsmodells angepasst werden sollten, es sollte sich also um eine suggestive Anleitung handeln. Die zweite Gestaltungsanforderung lautet daher wie folgt:

- DR2: Die Validierung des Geschäftsmodells sollte durch ein DSS unterstützt werden, das eine suggestive Anleitung für die konkreten zukünftigen Maßnahmen bietet.

Drittens sollte das DSS vom Benutzer lernen und eine Anleitung auf Nachfrage der Benutzer erstellen, da eine Geschäftsmodell-Validierung sehr unsicher und dynamisch ist und kein Angebot von vordefinierten Anleitungen erlaubt. Es braucht also eine dynamische Anleitung. Hieraus leiten wir die dritte Gestaltungsanforderung ab:

- DR3: Die Geschäftsmodell-Validierung sollte durch ein DSS unterstützt werden, das dynamische Änderungen erlaubt und aus den Eingaben der Benutzer lernt.

Viertens sollten die Benutzer in der Lage sein, die Funktionsweise der angebotenen Anleitung $\mathrm{zu}$ bestimmen. Dynamische und partizipative Entscheidungshilfen sind besonders effektiv bei der Verbesserung der Entscheidungsqualität, des Lernens der Benutzer und der Entscheidungsleistung bei hochkomplexen Aufgaben wie der Validierung von Geschäftsmodellen (z. B. Parikh et al. 2001, S. 303 f.). Daher formulieren wir die vierte Gestaltungsanforderung wie folgt: 
- DR4: Die Geschäftsmodell-Validierung sollte durch ein DSS unterstützt werden, das die Beteiligung der Nutzer (d. h. der Mentoren) ermöglicht.

Fünftens sollte das DSS dem Gründer zusätzliches Wissen zur Verfügung stellen, um ihm Hilfestellung bei der Verbesserung des Geschäftsmodells zu geben. Dementsprechend formulieren wir die fünfte Gestaltungsanforderung:

- DR5: Die Validierung des Geschäftsmodells sollte durch ein DSS unterstützt werden, das dem Nutzer prädiktives und präskriptives Wissen über das Geschäftsmodell zur Verfügung stellt.

Sechstens ist es von zentraler Bedeutung, dass der Benutzer langfristig aus den Aktionen lernt. Die sechste Gestaltungsanforderung lautet daher:

- DR6: Die Validierung des Geschäftsmodells sollte durch ein DSS unterstützt werden, das es dem Benutzer ermöglicht, aus Iterationen zu lernen.

In früheren Studien erwies sich die Kombination dieser sechs Dimensionen als am besten geeignet, um Einschränkungen bei der individuellen Entscheidungsfindung zu überwinden (Montazemi et al. 1996, S. 181 ff.; Parikh et al. 2001, S. 303 f.).

Schließlich muss dem Benutzer die Entscheidungshilfe so präsentiert werden, dass er daraus auf einfache Weise Schlussfolgerungen ziehen kann. Dafür ist eine Kombination verschiedener Präsentationsformate optimal, wie z. B. textbasiert und multimedial (Gregor \& Benbasat 1999, S. 497 ff.). Die Formate sollten den Charakteristika der Aufgabenstellung (d.h. der Gestaltung des Geschäftsmodells) entsprechen. Hieraus leiten wir die folgende Gestaltungsanforderung ab:

- DR7: Die Geschäftsmodell-Validierung sollte durch ein DSS unterstützt werden, das dem Benutzer eine Visualisierung der Anleitung bietet.

\subsubsection{Umsetzung von Entscheidungshilfen in unsicheren Kontexten}

Um die Entscheidungsfindung zu unterstützen, ist es wichtig, dem Benutzer eine qualitativ hochwertige Anleitung zu geben (Gregor \& Benbasat 1999, S. $497 \mathrm{ff}$; Silver 1991, S. 105 ff.). Zwei in jüngster Zeit populäre Ansätze für die Bereitstellung qualitativ hochwertiger Beratung bei der Entscheidungsunterstützung in unsicheren Umgebungen sind statistische Methoden (z. B. Creamer et al. 2016, S. 105 f.) und kollektive Intelligenz (Nagar \& Malone 2011, S. 6 ff.). Computerbasierte Methoden sind aufgrund der Fortschritte im Bereich des maschinellen Lernens und der maschinellen Intelligenz besonders wertvoll geworden, um verschiedene Formen von Daten aus unter- 
schiedlichen Quellen zu identifizieren, zu extrahieren und zu verarbeiten, um Vorhersagen im Zusammenhang mit Finanzierungsentscheidungen (Yuan et al. 2016, S. 67 ff.), finanziellen Erträgen (Creamer et al. 2016, S. 105 ff.) und dem Konkurs von Unternehmen (Olson et al. 2012, S. 464 ff.) zu treffen. Statistische Modelle sind unvoreingenommen, nicht durch kognitive Ressourcenbeschränkungen eingeschränkt und damit in der Lage, empirische Evidenz konsistent zu integrieren und optimal zu gewichten (Blattberg \& Hoch 1990, S. 880 ff.).

Maschinelles Lernen ist ein Paradigma, das es einem Computerprogramm (d. h. einem Algorithmus) ermöglicht, aus Erfahrungen (d. h. Daten) zu lernen und so die Leistung des Programms (z. B. die Genauigkeit) bei der Ausführung einer bestimmten Klasse von Aufgaben (z. B. Klassifikation oder Regression) zu verbessern. Folglich ist die maschinelle Intelligenz in der Lage, statistische Schlüsse auf der Grundlage von Mustern zu ziehen, die in zuvor gesehenen Fällen identifiziert wurden, und mit zunehmendem Dateninput zu lernen (Jordan \& Mitchell 2015, S. 255 ff.). Darüber hinaus ermöglichen solche Verfahren die Identifizierung komplexer Muster in Geschäftsmodellkonfigurationen und der Wechselbeziehungen zwischen einzelnen Komponenten und erweitern damit Methoden wie Geschäftsmodellsimulationen und Finanzszenarien.

Obwohl Techniken des maschinellen Lernens im Allgemeinen geeignet sein mögen, die Folgen bestimmter Entscheidungen bei der Gestaltung von Geschäftsmodellen auf der Grundlage früherer Datenverteilungen leicht quantifizierbarer Merkmale (z. B. Firmenalter, Teamgröße) vorherzusagen, sind sie oft auch verzerrt und versagen in sehr unsicheren Situationen, wenn sich beispielsweise die Daten im Laufe der Zeit verschieben und die Daten, die früher zum Training des Modells verwendet wurden, nicht mehr repräsentativ sind oder Muster auftreten, die der Algorithmus noch nie gesehen hat (Attenberg et al. 2011, S. 2 ff.; Dellermann et al. 2017b, S. 5 ff.). Darüber hinaus sind sie nicht in der Lage, subjektive Kriterien wie die Innovationsfähigkeit eines Wertangebots, die Vision oder den Fit des Teams oder die Gesamtkonsistenz eines Geschäftsmodells vorherzusagen (Cheng \& Bernstein 2015, S. 600 ff.). Aufgrund dieser Einschränkungen erfordern maschinelle Intelligenzsysteme eine Erweiterung durch die menschliche Intuition, um den Entwurf von Geschäftsmodellen erfolgreich zu leiten. Menschliche Entscheidungsträger bringen mehrere Vorteile mit sich. Der Mensch ist immer noch der Goldstandard für die Bewertung von Daten, die sich nicht leicht annotieren lassen (Baer \& McKool 2014, S. 81 ff.). Der Mensch ist besonders gut in der Lage, subjektive Beurteilungen von Daten zu liefern, die mit statistischen Verfahren objektiv schwer zu messen sind (Cheng \& Bernstein 2015, S. 600 ff.). Darüber hinaus verfügen menschliche Experten über ein hoch organisiertes Fachwissen, das es ihnen ermöglicht, sehr seltene Informationen zu erkennen und zu interpretieren. Solche Daten können zu spezifischen Ergebnissen führen, die a priori schwer vorherzusagen sind und eher Ausreißer in einem statistischen Modell darstellen würden (Blattberg \& Hoch 1990, S. 887 f.). Der Einsatz von Menschen zur Steigerung der maschinellen Intelligenz hat sich auch in vielen anderen Bereichen als wertvoll erwiesen (Kamar 2016, S. 4070 f.). Im Kontext der vorliegenden Forschung erwies sich der Einsatz der mensch- 
lichen Intuition als eine wertvolle Strategie, um den Erfolg von Geschäftsmodellen in der Frühphase zu antizipieren (Huang \& Pearce 2015, S. 638 ff.). Zwar haben individuelle Menschen immer noch die in den vorangegangenen Kapiteln besprochenen kognitiven Einschränkungen, doch können diese durch den Mechanismus der kollektiven Intelligenz minimiert werden (Larrick et al. 2011, S. 227 ff.). Dieser Ansatz aggregiert die Urteile einer größeren Gruppe von Menschen, um die Verzerrung individueller Bewertungen zu reduzieren (Blohm et al. 2016, S. 27 ff.; Leimeister et al. 2009, S. 197 ff.). Für die Beurteilung in unsicheren Situationen lässt sich der Wert von kollektiver Intelligenz gegenüber einzelnen Experten durch zwei Grundprinzipien erklären: Fehlerreduktion und Wissensaggregation (Larrick et al. 2011, S. 227 ff.). Während ein einzelner Entscheidungsträger anfällig für Verzerrungen und Fehler sein kann (wie z. B. einzelne Unternehmer oder Mentoren in unserem Kontext), minimiert das Prinzip der statistischen Aggregation solche Fehler durch die Kombination mehrerer Urteile. Darüber hinaus ist die Aggregation des Urteils mehrerer Personen aufschlussreich, da sie heterogenes Wissen über ein bestimmtes Problem aggregiert und die Erfassung eines umfassenderen Verständnisses eines Entscheidungsproblems ermöglicht (Soukhoroukova et al. 2012, S. 100 ff.; Keuschnigg \& Ganser 2016, S. 818 ff.; Ebel et al. 2016, S. 519 ff.).

Folglich argumentieren wir, dass die kollektive Intelligenz eine geeignete Methode darstellt, um Systeme des maschinellen Lernens zu erweitern, indem sie auf vielfältigeres Domänenwissen zugreift, es in einen Algorithmus integriert und die Gefahr einer voreingenommenen Interpretation verringert. Aufgrund dieser komplementären Fähigkeiten haben wir uns entschieden, maschinelle und kollektive Intelligenz zu kombinieren, um Gründern eine Entscheidungshilfe bei der Gestaltung ihres Geschäftsmodells zu geben. Wir bezeichnen solche kombinierten Systeme als Hybrid Intelligence Decision Support Systems (HIDSS). Folglich definieren wir HIDSS als computergestützte Entscheidungshilfe, um die Ergebnisse der Entscheidungsfindung eines Individuums zu verbessern, indem die sich ergänzenden Fähigkeiten von Mensch und Maschine kombiniert werden, um gemeinsam überlegene Ergebnisse zu erzielen und sich kontinuierlich zu verbessern, indem man voneinander lernt.

HIDSS könnte aus drei Gründen besonders geeignet sein, das vorliegende Problem zu lösen. Erstens sind Maschinen besser in der Lage, analytische Informationen zu verarbeiten und konsistente Ergebnisse zu liefern, insbesondere wenn die Daten verstreut und unstrukturiert sind (Einhorn 1972, S. 86 ff.). Im Zusammenhang mit der Validierung von Geschäftsmodellen sind dies unstrukturierte Daten hinsichtlich der Marktanforderungen, der technologischen Entwicklungen usw. Zweitens sind menschliche Entscheidungsträger besonders nützlich bei der Interpretation und Bewertung von weichen Informationen, da sie bei der Beurteilung von Faktoren wie Kreativität oder Zukunftsvorstellungen (die für die Gestaltung von Geschäftsmodellen erforderlich sind) oder bei der Bereitstellung umfassender Leitlinien für die zu ergreifenden Maßnahmen überlegen sind (Colton et al. 2011, S. 90 ff.). Drittens können Menschen in sehr unsicheren und komplexen Situationen, wie z. B. beim Aufbau eines Geschäftsmodells für ein neues Unternehmen, ihre Intuition und ihr Bauchgefühl einsetzen, was 
die statistischen Methoden ergänzt (Dellermann et al. 2018, S. 13). Dabei wird die kollektive Intelligenz verwendet, um die Vorteile des Menschen zu nutzen und gleichzeitig die Nachteile einzelner Entscheidungsträger, wie Verzerrungen oder Zufallsfehler, zu minimieren (Larrick et al. 2011, S. 227 ff.).

\subsection{Entwurf und Entwicklung (Phase 3)}

\subsubsection{Form- und Funktionsprinzipien für ein hybrides Entscheidungsunterstützungssystem (HIDSS)}

Um das HIDSS zu entwickeln, haben wir die erforderlichen Arten von Entscheidungshilfen in Designprinzipien (Prinzipien von Form und Funktion) übersetzt und dabei Mechanismen des maschinellen Lernens und der kollektiven Intelligenz kombiniert. Dieser Übersetzungsprozess ist in Abb. 8.2 dargestellt.

Um das HIDSS anzuwenden, müssen Unternehmer ihre impliziten Annahmen sowohl auf menschliche Mentoren als auch auf den Algorithmus des maschinellen Lernens übertragen, um ein gemeinsames Verständnis zu schaffen. Geschäftsmodelle sind mentale Repräsentationen der individuellen Überzeugungen eines Unternehmers, die explizit gemacht werden sollten, indem sie in ein digitales Objekt übertragen werden (Bailey et al. 2012, S. 1485 ff.; Carlile 2002, S. 442). Ansätze zur Übertragung solchen Wissens in eine gemeinsame Syntax sind erforderlich, um das Wissen sowohl für Menschen als auch für Algorithmen lesbar zu machen (Nonaka \& von Krogh 2009, S. 635 ff.).

Dabei können Ontologien genutzt werden, um den Wissensaustausch durch ein System von Vokabularen zu fördern. Solche Ontologien stellen beliebte Lösungen

Identifizierte Anforderungen

\begin{tabular}{|c|}
\hline DR1: Informative Anleitung \\
\hline DR2: Suggestive Anleitung \\
\hline DR3: Wissens-Aggregation \\
\hline DR4: Partizipative Gestaltung \\
\hline $\begin{array}{l}\text { DR5: Ressourceneffizientes } \\
\text { Feedback }\end{array}$ \\
\hline DR6: Gegenseitiges Lernen \\
\hline DR7: Visualisierung \\
\hline
\end{tabular}

Abgeleitete Gestaltungsprinzipien
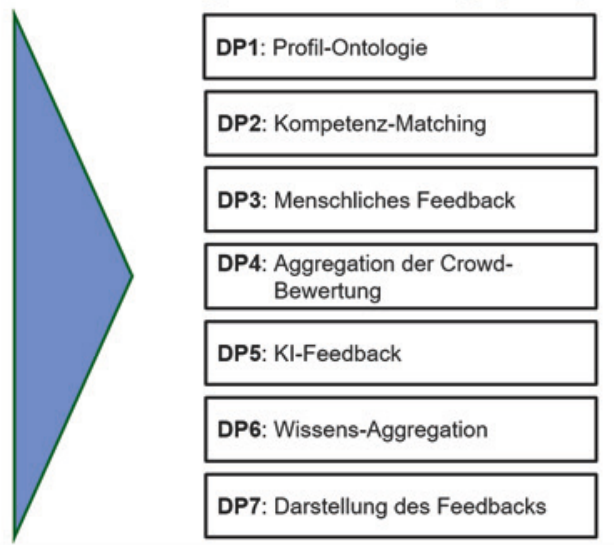

Abb. 8.2 Übertragung der Designanforderungen in Designprinzipien 
im Kontext von Geschäftsmodellen dar und umfassen Beschreibungen der zentralen Dimensionen eines Geschäftsmodells, wie z. B. Wertbeitrag, Wertschöpfung und Werterfassungsmechanismen (Osterwalder et al. 2005, S. 18). Dies ermöglicht es dem Benutzer, dynamisch und partizipativ Input in das DSS zu liefern (DR3 und DR4). Frühere Arbeiten zur menschlichen Kognition haben gezeigt, dass die Repräsentation von Wissen in einem solchen Objekt (d. h. die digitale Repräsentation des Geschäftsmodells) zur entsprechenden Aufgabe (d.h. zur Beurteilung des Geschäftsmodells) passen sollte, um die Qualität der menschlichen Entscheidung zu verbessern (John \& Kundisch 2015, S. 1 ff.). Da das Beurteilen eines Geschäftsmodells eine komplexe Aufgabe ist, ist eine visuelle Darstellung am besten geeignet, da sie kognitive Verfahren zur Maximierung der Entscheidungsqualität erleichtert (Speier \& Morris 2003, S. 1). Dies ermöglicht es menschlichen Bewertern, das Geschäftsmodell zu visualisieren. Um das Geschäftsmodell für den Algorithmus des maschinellen Lernens lesbar zu machen, erfordert die Ontologie auch ein maschinenlesbares Format, das durch die Standardisierung der Darstellung von Designentscheidungen (z. B. als Muster) oder die Verarbeitung natürlicher Sprache erreicht werden kann (John 2016, S. 9). Daher schlagen wir als erstes Entwurfsprinzip (Design Principle, DP) vor:

- DP1: Das Hybrid Intelligence DSS sollte mit einer ontologiebasierten Darstellung ausgestattet werden, um die Annahmen eines Unternehmers zu übertragen und ein gemeinsames Verständnis zwischen den Mentoren, der Maschine und dem Unternehmer zu schaffen.

Die bisherige Literatur zeigt, dass ein Bewerter, der für die Bereitstellung von Entscheidungshilfen zur Validierung von Geschäftsmodellen qualifiziert ist, auch ein Experte im jeweiligen Kontext ist (Ozer 2009, S. 1340 ff.). Eine solche Eignung führt zu einer höheren Fähigkeit, wertvolles Feedback zu geben, und ermöglicht die Vorhersage des potenziellen künftigen Erfolgs eines Geschäftsmodells auch in hochdynamischen Kontexten. Um als Bewerter geeignet zu sein und eine genauere Anleitung zu geben, sollte ein individueller menschlicher Mentor daher zwei Arten von Fachwissen besitzen: nachfrage- und angebotsseitiges Wissen (Ebel et al. 2016, S. 519 ff.). Nachfrageseitiges Wissen ist notwendig, um die Marktseite eines Geschäftsmodells zu verstehen (z. B. Kunden, Wettbewerber, Vertriebskanäle, Wertversprechen), was auf die Attraktivität eines Geschäftsmodells hinweist. Angebotsseitiges Wissen besteht aus Wissen über die Machbarkeit (z. B. Ressourcen, Aktivitäten) und die Rentabilität (z. B. Kostenstruktur, Ertragsmodell) einer Geschäftsmodellkonfiguration (Osterwalder et al. 2005, S. 1 ff.). Folglich ist es für ein HIDSS von zentraler Bedeutung, bestimmte Geschäftsmodelle mit spezifischen Fachexperten abzustimmen, um eine hohe Qualität der menschlichen Entscheidung zu gewährleisten (DR3 und DR4).

Das zweite Gestaltungsprinzip lautet daher wie folgt: 
- DP2: Das Hybrid Intelligence DSS sollte mit einem Empfehlungssystem für potenzielle Experten ausgestattet werden, damit der Unternehmer Zugang zu Expertise erhält.

Um den Unternehmern eine Orientierung zu geben, braucht es angemessene FeedbackMechanismen, um die entwickelten Annahmen zu bewerten (Blohm u. a. 2016, S. 3 f.). Aus der Perspektive der Verhaltensentscheidung kann dieses Feedback als eine Beurteilungsaufgabe kategorisiert werden, bei der eine endliche Menge von Alternativen (d. h. Geschäftsmodelldimensionen) mithilfe eines definierten Satzes von Kriterien beurteilt wird, wobei jede Alternative individuell mit Hilfe von Ratingskalen bewertet wird. Die Verwendung von Multi-Attribut-Ratingskalen für die Beurteilung und damit die Bereitstellung von Informationen ist in diesem Zusammenhang am besten geeignet (Riedl et al. 2013, S. 12). Die Multi-Attribut-Ratingskalen sollten die Erwünschtheit, Machbarkeit und Rentabilität eines Geschäftsmodells abdecken, indem sie Dimensionen bewerten, die starke Prädiktoren für den zukünftigen Erfolg sind, wie der Markt, die Geschäftsgelegenheit, das Unternehmerteam und die Ressourcen (Song et al. 2008, S. 13) (DR1). Darüber hinaus sollte der menschliche Mentor in der Lage sein, ein qualitatives Feedback zu geben, um das zukünftige Handeln des Unternehmers zu lenken und mögliche Weiterentwicklungen aufzuzeigen. Dies ermöglicht es dem Mentor, zusätzliche suggestive Hinweise zu geben (Silver 1991, S. 105 ff.) (DR2). Hieraus leiten wir das dritte Gestaltungsprinzip ab:

- DP3: Versehen Sie das Hybrid Intelligence DSS mit qualitativen und quantitativen Feedback-Mechanismen, um den Mentor in die Lage zu versetzen, ein angemessenes Feedback zu geben.

Darüber hinaus kann der menschliche Input auch dazu verwendet werden, einen Klassifikator für das maschinelle Lernen zu trainieren, der die Wahrscheinlichkeit bewertet, dass innerhalb der Version des vorgestellten Geschäftsmodells Meilensteine erreicht werden. Dieses Verfahren ermöglicht eine konsistente Verarbeitung und Gewichtung des kollektiven menschlichen Urteils, wie es erforderlich ist, um eine qualitativ hochwertige Bewertung durch kollektive Intelligenz zu erreichen (Keuschnigg \& Ganser 2016, S. 818 ff.) (DR1 und DR4). Deshalb formulieren wir das vierte Gestaltungsprinzip folgendermaßen:

- DP4: Bereitstellung eines Crowd-based Classifiers für das Hybrid Intelligence DSS, um die Ergebnisse von Geschäftsmodell-Designentscheidungen auf der Grundlage der menschlichen Beurteilung vorherzusagen.

Jedes erstellte Geschäftsmodell besteht aus verschiedenen Designentscheidungen (z. B. in Bezug auf Wertversprechen, Ertragsmodelle usw.). Dies ermöglicht es, überwachte Ansätze des maschinellen Lernens einzusetzen, um maschinelles Feedback bezüglich 
der Erfolgswahrscheinlichkeit eines bestimmten Geschäftsmodellelements zu liefern (Jordan \& Mitchell 2015, S. 256 ff.). Beaufsichtigte Algorithmen ermöglichen es einer Maschine, aus den Trainingsdaten (d. h. den Eingaben des Benutzers) zu lernen, um vorherzusagen, welche Konfiguration zu einem günstigen Ergebnis (d. h. zum Erreichen eines Meilensteins) führt (DR1). Zu diesem Zweck muss der Benutzer entsprechende Informationen bereitstellen, wenn ein Geschäftsmodell einen Meilenstein erreicht (z. B. Finanzierung). Dieses Verfahren ermöglicht es, die Fähigkeit der Maschine zur Bewertung neuer Geschäftsmodellkonfigurationen zu trainieren, um die Erfolgswahrscheinlichkeit einer bestimmten Geschäftsmodellversion vorherzusagen und so die Annahmen eines Unternehmers zu validieren (oder abzulehnen). Das fünfte Gestaltungsprinzip lautet daher:

- DP5: Versehen Sie das Hybrid Intelligence DSS mit einer maschinellen Rückkopplungsfähigkeit, um die Ergebnisse von Geschäftsmodellentwürfen auf der Grundlage einer statistischen Bewertung vorherzusagen.

Die Geschäftsmodell-Validierung ist ein iterativer Prozess, der aus der Validierung des bestehenden Modells, seiner Anpassung und der anschließenden Revalidierung besteht. Daher sollte das HIDSS die Ergebnisse jeder Validierungsrunde aggregieren, um zu zeigen, wie das menschliche und das maschinelle Feedback die Annahmen eines Unternehmers verändern und wie solche Änderungen zu einem bestimmten Ergebnis führen (John 2016, S. 16 f.). Die Anhäufung solchen Wissens kann kognitive Prozesse auslösen, die das Verständnis des Unternehmers für den Bereich umstrukturieren (Sengupta \& Abdel-Hamid 1993, S. 412 f.). Das von uns vorgeschlagene HIDSS muss das während der Nutzung geschaffene Wissen in einem Repository akkumulieren, die Beratungsqualität durch maschinelles Lernen kontinuierlich verbessern und nicht nur aus den Iterationen des individuellen Validierungsprozesses, sondern auch von anderen Nutzern des Systems (z. B. anderen Unternehmern) lernen (Jordan \& Mitchell 2015, S. 259 f.). Der Wissensspeicher kann dann allgemeine Muster dafür speichern, wie Änderungen in einem Geschäftsmodell von Menschen oder dem Algorithmus des maschinellen Lernens bewertet werden und wie sie zum Erreichen bestimmter Meilensteine führen (z. B. zum Erhalt von Finanzmitteln) (DR3, DR5 und DR6). Wir schlagen deshalb folgendes Gestaltungsprinzip vor:

- DP6: Bereitstellung eines Wissensaggregationsspeichers für das Hybrid Intelligence DSS, damit es aus dem Prozess lernen kann.

Schließlich müssen dem Unternehmer Lernprozesse sowie die Aufnahme von Wissen (in Form zusätzlicher Informationen) durch eine geeignete Darstellung der Anleitung ermöglicht werden, was am besten durch ein Dashboard erreicht werden kann (Benbasat et al. 1986, S. 1094 ff.). Um eine hohe Entscheidungsqualität für den Benutzer zu erreichen, sollte eine kognitive Verbindung zwischen der hochkomplexen Aufgabe (d. h. 
Entscheidungen über die Gestaltung des Geschäftsmodells zu treffen) und der Anleitung durch eine entsprechende visuelle Darstellung hergestellt werden (DR1, DR2, DR6 und DR7). Darüber hinaus sollte die Anleitung entlang der Geschäftsmodell-Dimensionen strukturiert werden, um dem Benutzer das Verstehen und Abrufen der Anleitung zu erleichtern (Baker et al. 2009, S. 533 ff.). Hieraus leiten wir das folgende Gestaltungsprinzip ab:

- DP7: Das Hybrid Intelligence DSS sollte mit einer visuellen Darstellung versehen werden, damit der Unternehmer Zugang zu informativen und suggestiven Anleitungen erhält.

\subsubsection{Implementierung des hybriden Entscheidungsunterstützungssystems}

Bei der Implementierung unseres HIDSS haben wir die identifizierten Designprinzipien in konkrete Designmerkmale überführt, die spezifische Charakteristika des Artefakts darstellen, um jedes der Designprinzipien zu berücksichtigen. Um unsere Designprinzipien in eine Prototyp-Version des HIDSS-Artefakts zu implementieren, haben wir einen Cloud-basierten Web-Service erstellt. Der Prototyp des Artefakts besteht aus einer grafischen Benutzeroberfläche (Graphical User Interface, GUI), die die Eingabe und Visualisierung des Geschäftsmodells des Unternehmers ermöglicht. Zu diesem Zweck haben wir eine Webanwendung in Angular entwickelt. Die Geschäftsmodelle werden in einem standardisierten und dynamisch anpassbaren Format dargestellt, das es dem Unternehmer ermöglicht, für jedes Element Entscheidungen entlang der Dimensionen Wertangebot, Wertlieferung, Wertschöpfung und Werterfassung zu treffen (Osterwalder \& Pigneur 2013, S. 237 ff.) (DP1). Die Zuordnung von Fachwissen wird durch eine einfache Markierung (d. h. Markt, Technologie oder Finanzen) erreicht. Diese Tags werden dann mit einer Liste kategorisierter Mentoren abgeglichen (DP2). Die Mechanismen, die ein menschliches Feedback ermöglichen, werden mithilfe desselben Werkzeugs implementiert. Dabei wurden Bewertungsskalen (1 bis 10) implementiert, die aus 21 Kriterien bestehen und die Erwünschtheit, Umsetzbarkeit, Skalierbarkeit und Rentabilität eines Geschäftsmodells abdecken. Darüber hinaus steht eine Textbox zur Verfügung, in der konkrete qualitative Hinweise zur Verbesserung des Geschäftsmodells eingegeben werden können. Diese Hinweise für das Feedback wurden in Bezug auf das Wertangebot, die Werterbringung, die Wertschöpfung und die Werterfassungsmechanismen des Geschäftsmodells (DP3) strukturiert. Um den Algorithmus des maschinellen Lernens mit ersten Daten zu trainieren, sammelten wir öffentlich zugängliche Informationen über Start-up-Geschäftsmodelle und ihren jeweiligen Erfolg.

Der maschinelle Lernteil des Prototyps wurde auf der Grundlage des Open-SourceFrameworks für maschinelles Lernen, TensorFlow, in der Programmiersprache Python entwickelt. Für den Crowd-basierten Klassifikator haben wir einen Klassifikations- und 
Regressionsbaum (CART) verwendet, da dieser sowohl eine gute Leistung als auch eine gute Interpretierbarkeit der Ergebnisse durch die Replikation menschlicher Entscheidungsstile bietet (Liaw \& Wiener 2002, S. 18 ff.) (DP4). Der gleiche Lernalgorithmus wurde zur Analyse der komplexen Interaktionen zwischen den Komponenten des Geschäftsmodells verwendet. Damit wird die Erfolgswahrscheinlichkeit eines bestimmten Geschäftsmodells berechnet (DP5). Alle Ergebnisse (d. h. Geschäftsmodellkomponenten, Profildaten von Mentoren und menschliches Urteilsvermögen) werden im JSON-Format in einer relationalen PostgreSQL-Datenbank auf einem Ubuntu-SSDServer (DP6) gespeichert. Die abschließende Visualisierung der Ergebnisse (informative und suggestive Anleitungen) wird durch das in Angular implementierte Dashboard bereitgestellt (DP7). Dieses stellt aggregierte Ergebnisse entlang der Dimensionen Erwünschtheit, Machbarkeit und Rentabilität sowie die vorhergesagte Erfolgswahrscheinlichkeit entlang der Ergebnisdimensionen Überleben und Finanzierung dar, die allgemein akzeptierte Proxies für erfolgreiche Geschäftsmodelle in der Frühphase darstellen. Die Architektur unseres Prototyp-Artefakts ist in Abb. 8.3 visualisiert.

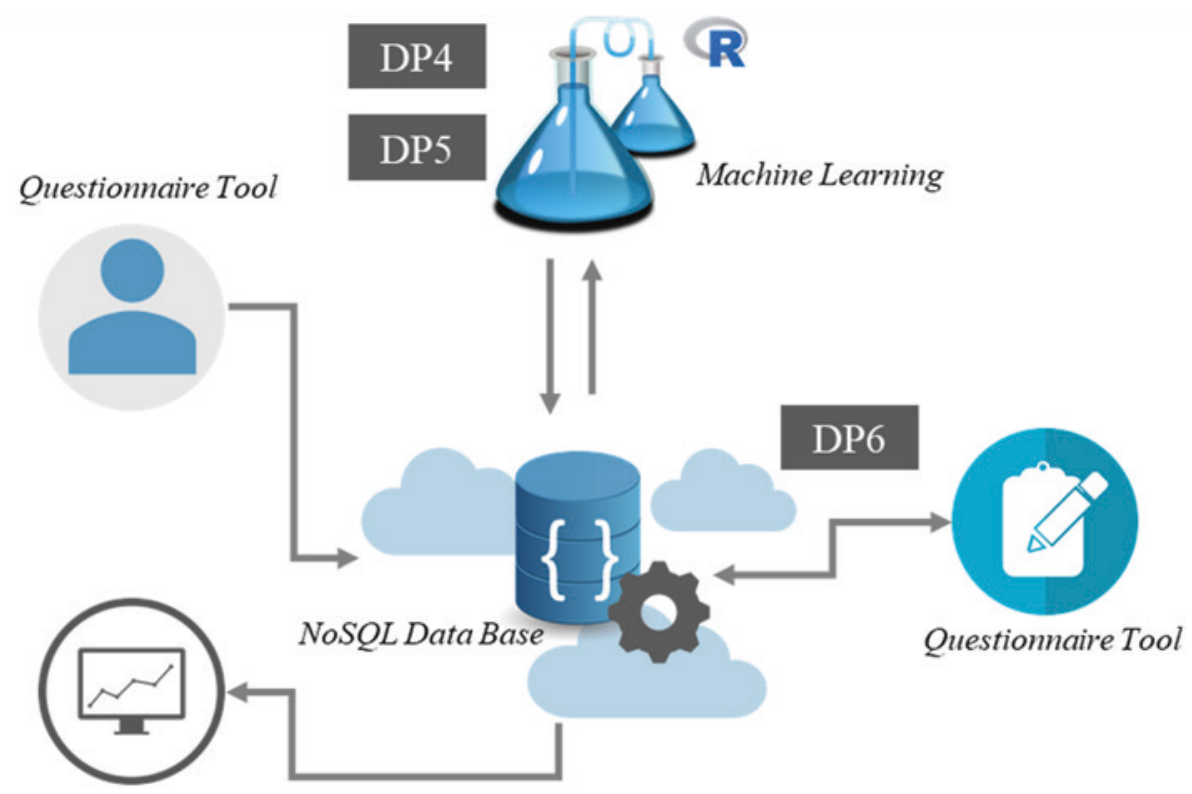

Business Intelligence Tool

Abb. 8.3 Architektur des Protoypen 


\subsection{Demonstration des Design-Artefakts (Phase 4)}

Die erste Evaluierung unseres HIDSS wurde im Sinne einer Ex-Ante-Intervention durchgeführt, um sicherzustellen, dass das IT-Artefakt als wirksames Instrument zur Lösung des zugrunde liegenden Problems konzipiert ist (Venable et al. 2016, S. 79). Zu diesem Zweck haben wir beschlossen, explorative Fokusgruppen einzusetzen, um das Artefaktdesign auf der Grundlage des Feedbacks der Teilnehmer zu verfeinern. In insgesamt acht Fokusgruppen wurden unsere Designprinzipien von Unternehmern und Mentoren sowie von Entwicklern demonstriert, validiert und verfeinert, um die technische Machbarkeit der Designprinzipien in einer naturalistischen Umgebung zu überprüfen. Während dieser Ex-Ante-Evaluierung konzentrierten wir uns auf die Klarheit, Vollständigkeit, interne Konsistenz und Anwendbarkeit zur Lösung des praktischen Problems. Die vorläufige Version der Entwurfsprinzipien wurde dann angepasst, bevor sie in unserem PrototypArtefakt instanziiert wurde.

Die erforderlichen Änderungen bezogen sich insbesondere auf die Anpassung der Fachkenntnisse und die Ontologie des Geschäftsmodells. Die Teilnehmer schlugen einen Wechsel von bekannten Geschäftsmodell-Visualisierungen (d. h. dem Business Model Canvas) zu einer neuartigen Form von Start-up-Profilen vor, da die Mentoren vertiefte Informationen über das jeweilige Start-up benötigen. Insbesondere bei einer IT-basierten und zeitlich-räumlich asynchronen Lösung ist ein solcher Ansatz zwingend erforderlich, um eine qualitativ hochwertige Beratung zu gewährleisten. Darüber hinaus ergab die Evaluierung die Notwendigkeit eines Expertenabgleichs auf einer feingranularen Ebene. Abgesehen vom Matching zwischen der Branche des Start-ups und dem jeweiligen Experten (z. B. FinTech-Start-up mit einem Experten aus der Bankenbranche) ist es entscheidend, auch zu jeder Dimension des Geschäftsmodells Feedback von einem entsprechenden Expertentyp zu erhalten (z. B. Finanzexperte zur Bewertung der Werterfassungsmechanismen). Schließlich baten die Teilnehmer des Workshops um die Möglichkeit, ein vertieftes qualitatives Feedback zu geben, das nicht nur Verbesserungsvorschläge wie z. B. die Änderung des vorgeschlagenen Ertragsmodells umfasst, sondern auch Hinweise zum weiteren Vorgehen und zum Erreichen des jeweiligen Ziels enthält.

\subsection{Evaluierung (Phase 5)}

Für die Ex-Post-Evaluierung der Umsetzung unserer instanziierten Designprinzipien in ein konkretes IT-Artefakt wurde eine qualitative Evaluierungsmethode verwendet, um die Anwendbarkeit im realen Kontext zu testen und die Durchführbarkeit, Effektivität, Effizienz und Zuverlässigkeit bei der Unterstützung von Entscheidungen beim Geschäftsmodellentwurf zu bewerten (Venable et al. 2016, S. 79). Insbesondere führten wir Workshops mit Entscheidungsträgern und potenziellen Anwendern unseres HIDSS in der Praxis durch. 
Wir wählten diesen Evaluierungsansatz aus mehreren Gründen: Erstens ermöglichte uns die Flexibilität der Methode, das Artefakt bei Bedarf anzupassen. Zweitens ermöglichte uns dieser Ansatz eine direkte Interaktion mit den potenziellen Nutzern des Systems, wodurch sichergestellt wurde, dass das Artefakt eindeutig verstanden wurde. Schließlich lieferte die Fokusgruppenmethode riesige Mengen reichhaltiger Daten, die ein tieferes Verständnis der Effektivität und Effizienz des Artefakts zur Lösung eines realen Problems in einer realen Geschäftsumgebung ermöglichten.

Für die Durchführung von insgesamt acht Fokusgruppen-Workshops rekrutierten wir 24 Teilnehmer (Mitarbeiter von Geschäftsinkubatoren und Acceleratoren sowie unabhängige Start-up-Mentoren). Vier der Fokusgruppen setzten sich aus Teilnehmern aus Gründerzentren, zwei aus Acceleratoren und zwei aus unabhängigen Mentoren zusammen. Wir stellten das HIDSS über einen Click-Through-Ansatz vor und erläuterten jedes der Designprinzipien im Detail. Anschließend ging es in den Workshops um die Effektivität der Lösung der realen Probleme. Wie die Ergebnisse unserer Evaluierung zeigen, überwindet das HIDSS die Grenzen bisheriger Lösungen, indem es die analytische Verarbeitung der Interaktion zwischen komplexen Geschäftsmodellmustern und den Input der menschlichen Intuition kombiniert. Die Fokusgruppen zeigen, dass die Entscheidungshilfe Unternehmer dabei unterstützt, mit der hochkomplexen und unsicheren Aufgabe umzugehen, Entscheidungen über die Gestaltung von Geschäftsmodellen zu treffen und ihre individuellen Grenzen zu überwinden. Darüber hinaus ermöglicht eine IT-basierte Lösung, die das kollektive Urteilsvermögen der verschiedenen Mentoren aggregiert, die Subjektivität zu reduzieren und gleichzeitig Wissen $\mathrm{zu}$ aggregieren, das durch maschinelles Lernen gespeichert werden kann. Schließlich erlaubt die hybride Natur unseres vorgeschlagenen Designs, mit weichen Faktoren und extremer Unsicherheit umzugehen, indem die menschliche Intuition in die FeedbackSchleife einbezogen wird. Darüber hinaus erleichtert der Einsatz von maschinellem Lernen, zur Identifizierung der komplexen Interaktion zwischen verschiedenen Geschäftsmodellelementen den Umgang mit der Komplexität von Start-up-Geschäftsmodellen.

Im Rahmen der summativen Ex-Post-Evaluierung wurde jedes Konstruktionsprinzip im Detail bewertet. Alle vorgeschlagenen Entwurfsprinzipien und ihre Instanziierungen wurden als nützlich und effektiv bei der Lösung der Probleme empfunden, die bei der Entscheidungsunterstützung für die Validierung von Geschäftsmodellen auftreten. Die Teilnehmer argumentierten, dass das HIDSS besonders geeignet sei, die Entscheidungsqualität und Effizienz der Gründer zu verbessern (DR6: Wissen) und ihnen beim Lernen zu helfen (DR5: Lernen). Der digitale Charakter des Tools wurde als zeit- und ressourcensparend empfunden und ermöglichte es den Mentoren, zeitund ortsunabhängig zu beraten und somit eine qualitativ hochwertige Beratung $\mathrm{zu}$ bieten (DR1: informativ und DR2: suggestiv). Die Führungskräfte von Gründerzentren und Acceleratoren begrüßten die Möglichkeit, Wissen über die Gestaltung von Geschäftsmodellen von Start-ups zu sammeln und dieses Wissen durch den Ansatz des maschinellen Lernens implizit zu teilen (DR3: dynamisch). Die Experten waren sich 
einig, dass dies die Überlebensrate neuer Unternehmen in einem frühen Stadium erhöhen könnte. Die Teilnehmer schätzten außerdem die Möglichkeit, externe Mentoren aus verschiedenen Branchen einzusetzen. Während die öffentlich finanzierten Inkubatoren die Anwendbarkeit in diesem Zusammenhang als sehr hoch einschätzten, schlug der gewinnorientierte Accelerator vor, dass für externe Mentoren über die intrinsische Belohnung hinaus Kompensationsmodelle definiert werden sollten. Obwohl altruistisches Mentoring in der Praxis gut funktioniert (z. B. Businessplan-Wettbewerbe und Feedback), sollte für die Anwendung unseres Artefakts in der Praxis ein Belohnungsmechanismus in Betracht gezogen werden. Darüber hinaus sehen die Experten ein großes Potenzial in der Verbesserung der Entscheidungshilfe durch maschinelles Lernen. Sie wiesen aber auch darauf hin, dass aufgrund der menschlichen Komponente des HIDSS die Akzeptanz bei den Unternehmern möglicherweise höher ist als bei rein statistischen Modellierungen und Simulationen (DR4: partizipative Beratung). Schließlich wurde das Dashboard zur Visualisierung der Entscheidungshilfe durch Grafiken und Feedback-Text als günstig empfunden, um die Entscheidungshilfe für Unternehmer leicht zugänglich zu machen (DR7: Visualisierung).

Die Ergebnisse der Evaluierung zeigen jedoch auch zwei Kritikpunkte auf, die vor dem Einsatz in einer realen Umgebung verbessert werden sollten. Zunächst hoben die Teilnehmer die Notwendigkeit hervor, Vertrauen in KI-basierte DSS zu schaffen. Zwar ist die Bereitstellung hochpräziser Entscheidungshilfen von entscheidender Bedeutung, doch geht es dabei immer um einen Kompromiss zwischen Genauigkeit und Transparenz, was von den meisten Teilnehmern hervorgehoben wurde. Zukünftige Forschungsarbeiten könnten diese Frage bei der Anwendung von HIDSS in Unternehmenskontexten untersuchen. Zweitens wiesen die Teilnehmer darauf hin, dass die Anwendung solcher IT-basierten Leitlinien möglicherweise als mangelnde persönliche Unterstützung durch die Mentoren empfunden werden könnte. Obwohl der Wert des HIDSS für alle Teilnehmer offensichtlich war, argumentierten sie, dass bei der Kommunikation mit den Nutzern solcher Systeme (d. h. mit Unternehmern und Mentoren) der Mensch im Mittelpunkt stehen und die maschinelle Intelligenz nur als Ergänzung betrachtet werden sollte.

\subsection{Fazit}

Unsere Ergebnisse implizieren, dass die Fähigkeiten der formalen Analyse und der Mustererkennung, die durch maschinelles Lernen bereitgestellt werden, in Kombination mit der menschlichen Intuition, die durch die kollektive Intelligenz bereitgestellt wird, eine wertvolle Lösung für den extrem unsicheren Kontext der iterativen Geschäftsmodell-Validierung in der Frühphase von Unternehmensgründungen darstellen. Ein HIDSS ermöglicht es Mentoren, die erforderliche Entscheidungshilfe zu geben, um Gründer bei ihren Entscheidungen zu unterstützen. Dafür gibt es mehrere Gründe. Erstens erfassen die von uns vorgeschlagenen Designprinzipien die komplexe Wechsel- 
wirkung zwischen den Entscheidungen in Bezug auf die Gestaltung von Geschäftsmodellen und der dynamischen Natur solcher Entscheidungen und überwinden so die Grenzen traditioneller Analysemethoden wie Modellierung und Simulation (Haaker et al. 2017, S. 14 ff.; Euchner \& Ganguly 2014, S. 33 ff.). Unsere Ergebnisse bieten damit auch einen neuartigen und innovativen Ansatz, der im Einklang ist mit früheren Forschungen über Dynamik und komplexe Wechselwirkungen (Möllers et al. 2017, S. 125 ff.). Zweitens ergänzt das HIDSS traditionelle Analysemethoden um die menschliche Intuition. Durch die Nutzung der kollektiven Intelligenz anstelle von individuellen Entscheidungsträgern vermeidet unser Ansatz die Einschränkungen individueller Mentoren. Folglich profitiert das HIDSS von dem heterogenen Wissen mehrerer Experten und aggregiert die Bewertungen einer größeren Gruppe, um die in individuellen Urteilen inhärente Verzerrung zu reduzieren. Dieses Verfahren ist in dem unsicheren und komplexen Kontext der Unterstützung von Entscheidungen bei der Geschäftsmodellgestaltung besonders wertvoll, da es nicht nur informative Anleitungen in Form von Geschäftsmodellbewertungen gibt, sondern auch suggestive Anleitungen liefert, die Unternehmer auf direkte Interventionen zur Verbesserung des Geschäftsmodells hinweisen. Drittens speichert das HIDSS das geschaffene Wissen in einem Wissensspeicher. Auf lange Sicht ermöglicht dies sowohl Unternehmern als auch Mentoren, von den Erfahrungen anderer zu lernen. Im Laufe seiner Anwendung lernt das System, welche Entscheidungen in Bezug auf die Geschäftsmodellgestaltung von Menschen positiv und negativ bewertet werden und welche Entscheidungen in einem bestimmten Kontext zu einem bestimmten Ergebnis führen. Dies kann in Zukunft die vollständige Automatisierung solcher Entscheidungshilfen ermöglichen. Viertens bietet die digitale Natur des HIDSS eine Möglichkeit, das menschliche Mentoring zu digitalisieren. Solche IT-Systeme können ein Geschäftsmodell iterativ validieren sowie asynchrones und ortsunabhängiges Feedback für ein ressourceneffizientes Mentoring ermöglichen.

Schließlich weisen unsere Ergebnisse darauf hin, dass diese neue Klasse von DSS besonders in sehr unsicheren Kontexten wertvoll sein könnte. Mit zunehmender Unsicherheit nehmen die relativen Vorteile statistischer Methoden bei der Bereitstellung von Entscheidungshilfen ab und der Wert der menschlichen Intuition nimmt zu. Da die Kombination von formaler Analyse und Intuition bei Vorhersagen in extrem unsicheren Kontexten allgemein akzeptiert wird (Huang \& Pearce 2015, S. 634 ff.), kann ein HIDSS eine hochwertige Orientierungshilfe bieten, die auch in anderen Bereichen wie Innovation oder Medizin funktionieren könnte. Die von uns vorgeschlagenen Designprinzipien stellen einen ersten Schritt in diese Richtung dar. Abb. 8.4 zeigt die vorgeschlagenen Designprinzipien für ein HIDSS in einer schematischen Darstellung des verwendeten Arbeitsablaufs. 


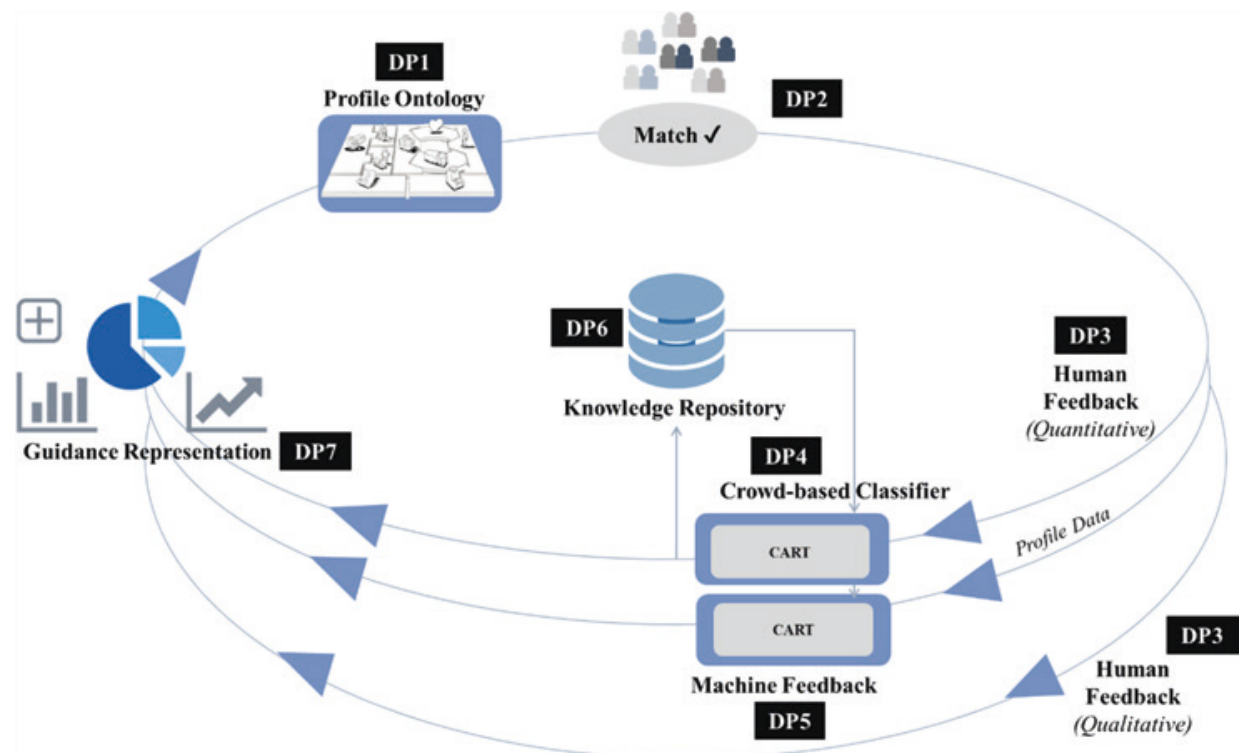

Abb. 8.4 Zusammenfassung der Designprinzipien für ein HIDSS

\section{Literatur}

Aerts, K., Matthyssens, P., \& Vandenbempt, K. (2007). Critical role and screening practices of European business incubators. Technovation, 27(5), 254-267.

Al-Debei, M. M., \& Avison, D. (2010). Developing a unified framework of the business model concept. European Journal of Information Systems, 19(3), 359-376.

Alvarez, S. A., \& Barney, J. B. (2007). Discovery and creation: Alternative theories of entrepreneurial action. Strategic Entrepreneurship Journal, 1(1-2), 11-26.

Alvarez, S. A., Barney, J. B., \& Anderson, P. (2013). Forming and exploiting opportunities: The implications of discovery and creation processes for entrepreneurial and organizational research. Organization Science, 24(1), 301-317.

Arnold, V., Collier, P. A., Leech, S. A., \& Sutton, S. G. (2004). Impact of intelligent decision aids on expert and novice decision-makers' judgments. Accounting and Finance, 44(1), 1-26.

Attenberg, J., Ipeirotis, P. G., \& Provost, F. (2011). Beat the machine: Challenging workers to find the unknown unknowns. In Proceedings of the 11th AAAI conference on human computation (S. 2-7).

Baer, J., \& McKool, S. S. (2014). The gold standard for assessing creativity. International Journal of Quality Assurance in Engineering and Technology Education (IJQAETE), 3(1), 81-93.

Bailey, D. E., Leonardi, P. M., \& Barley, S. R. (2012). The lure of the virtual. Organization Science, 23(5), 1485-1504.

Baker, J., Jones, D. R., \& Burkman, J. (2009). Using visual representations of data to enhance sensemaking in data exploration tasks. Journal of the Association for Information Systems, 10(7), 533. 
Benbasat, I., Dexter, A. S., \& Todd, P. (1986). An experimental program investigating color-enhanced and graphical information presentation: An integration of the findings. Communications of the ACM, 29(11), 1094-1105.

Bharadwaj, A., El Sawy, O., Pavlou, P., \& Venkatraman, N. (2013). Digital Business Strategy: Toward a Next Generation of Insights. MIS Quarterly, 37(2), 471-482. Retrieved June 9, 2021, from http://www.jstor.org/stable/43825919.

Blank, S. (2013). Why the lean start-up changes everything. Harvard Business Review, 91(5), 63-72.

Blattberg, R. C., \& Hoch, S. J. (1990). Database models and managerial intuition: $50 \%$ model + 50\% manager. Management Science, 36(8), 887-899.

Blohm, I., Riedl, C., Füller, J., \& Leimeister, J. M. (2016). Rate or trade? Identifying winning ideas in open idea sourcing. Information Systems Research, 27(1), 27-48.

Bøllingtoft, A., \& Ulhøi, J. P. (2005). The networked business incubator - Leveraging entrepreneurial agency? Journal of Business Venturing, 20(2), 265-290.

Bouwman, H., Zhengjia, M., van der Duin, P., \& Limonard, S. (2008). A business model for IPTV service: A dynamic framework. info, 10(3), 22-38.

Carlile, P. R. (2002). A pragmatic view of knowledge and boundaries: Boundary objects in new product development. Organization Science, 13(4), 442-455.

Cavalcante, S., Kesting, P., \& Ulhøi, J. (2011). Business model dynamics and innovation: (Re) establishing the missing linkages. Management Decision, 49(8), 1327-1342.

Cheng, J., \& Bernstein, M. S. (2015). Flock: Hybrid crowd-machine learning classifiers. In Proceedings of the 18th ACM conference on computer supported cooperative work \& social computing, February 2015 (S. 600-611).

Cohen, S. G., \& Hochberg, Y. V. (2014). Accelerating startups: The seed accelerator phenomenon. SSRN Journal, 2014, 1-16.

Colton, S., Charnley, J., \& Pease, A. (2011). Computational creativity theory: The FACE and IDEA descriptive models. In 2nd International Conference on Computational Creativity (S. 90-95).

Creamer, G. G., Ren, Y., Sakamoto, Y., \& Nickerson, J. V. (2016). A textual analysis algorithm for the equity market: The European case. The Journal of Investing, 25(3), 105-116.

Daas, D., Hurkmans, T., Overbeek, S., \& Bouwman, H. (2013). Developing a decision support system for business model design. Electronic Markets, 23(3), 251-265.

Dellermann, D., Lipusch, N., \& Ebel, P. A. (2017a). Developing design principles for a crowdbased business model validation system. In A. Maedche, J. Vom Brocke, \& A. Hevner (Hrsg.), Designing the digital transformation. Proceedings of the 12th international conference DESRIST 2017, Karlsruhe, Germany, May 30-June 1, 2017 (S. 163-178). Springer International Publishing. https://doi.org/10.1007/978-3-319-59144-5_10.

Dellermann, D., Lipusch, N., Ebel, P. A., Popp, K. M., \& Leimeister, J. M. (2017b). Finding the unicorn: Predicting early stage startup success through a hybrid intelligence method. In Proceedings ICIS 2017, Seoul.

Dellermann, D., Lipusch, N., \& Ebel, P. A. (2018). Heading for new shores: Crowdsourcing for entrepreneurial opportunity creation. In: Proceeding of the European Conference on Information Systems.

Demil, B., \& Lecocq, X. (2010). Business model evolution: In search of dynamic consistency. Business Models, 43(2), 227-246.

Demil, B., Lecocq, X., Ricart, J. E., \& Zott, C. (2015). Introduction to the SEJ special issue on business models: Business models within the domain of strategic entrepreneurship. Strategic Entrepreneurship Journal, 9(1), 1-11. 
Ebel, P., Bretschneider, U., \& Leimeister, J. M. (2016). Leveraging virtual business model innovation: A framework for designing business model development tools. Information Systems Journal, 26(5), 519-550.

Einhorn, H. J. (1972). Expert measurement and mechanical combination. Organizational Behavior and Human Performance, 7(1), 86-106.

Euchner, J., \& Ganguly, A. (2014). Business model innovation in practice. Research-Technology Management, 57(6), 33-39.

Gönül, M. S., Önkal, D., \& Lawrence, M. (2006). The effects of structural characteristics of explanations on use of a DSS. Decision Support Systems, 42(3), 1481-1493.

Gordijn, J., Akkermans, H., \& Van Vliet, J. (2001). Designing and evaluating e-business models. IEEE Intelligent Systems, 16(4), 11-17.

Gregor, S., \& Benbasat, I. (1999). Explanations from intelligent systems: Theoretical foundations and implications for practice. Management Information Systems Quarterly, 23(4), 497-530.

Gregor, S., \& Hevner, A. (2013). Positioning and Presenting Design Science Research for Maximum Impact. MIS Quarterly, 37(2), 337-355. Retrieved June 9, 2021, from http://www. jstor.org/stable/43825912.

Haaker, T., Bouwman, H., Janssen, W., \& de Reuver, M. (2017). Business model stress testing: A practical approach to test the robustness of a business model. Futures, 89, 14-25.

Hevner, A. R. (2007). A three-cycle view of design science research. Scandinavian Journal of Information Systems, 19(2), 4.

Huang, L., \& Pearce, J. L. (2015). Managing the unknowable: The effectiveness of early-stage investor gut feel in entrepreneurial investment decisions. Administrative Science Quarterly, 60(4), 634-670.

John, T. (2016). Supporting Business Model Idea Generation Through Machine-generated Ideas: A Design Theory. In Proceedings of the 37th International Conference on Information Systems (ICIS), Dublin, Ireland. Dublin, Ireland.

John, T., \& Kundisch, D. (2015). in: Proceedings of the 36th International Conference on Information Systems (ICIS), Fort Worth, USA.

Jordan, M. I., \& Mitchell, T. M. (2015). Machine learning: Trends, perspectives, and prospects. Science, 349(6245), 255-260.

Kamar, E. (2016). Directions in hybrid intelligence: Complementing AI systems with human intelligence. In Proceedings of the twenty-fifth international joint conference on artificial intelligence (S. 4070-4073).

Keuschnigg, M., \& Ganser, C. (2016). Crowd wisdom relies on agents' ability in small groups with a voting aggregation rule. Management Science, 63(3), 818-828.

Larrick, R. P. and Feiler, D. C. (2015). Expertise in Decision Making. In: The Wiley Blackwell Handbook of Judgment and Decision Making (S. 696-721) (eds G. Keren and G. Wu). https:// doi.org/10.1002/9781118468333.ch24.

Larrick, R. P., Mannes, A. E., Soll, J. B., \& Krueger, J. I. (2011). The social psychology of the wisdom of crowds. In J. I. Krueger (Hrsg.), Social judgment and decision making (S. 227-242). Taylor \& Francis.

Leimeister, J. M., Huber, M., Bretschneider, U., \& Krcmar, H. (2009). Leveraging crowdsourcing: Activation-supporting components for IT-based ideas competition. Journal of Management Information Systems, 26(1), 197-224.

Liaw, A., \& Wiener, M. (2002). Classification and regression by random forest. $R$ News, 2(3), $18-22$.

Limayem, M., \& DeSanctis, G. (2000). Providing decisional guidance for multicriteria decision making in groups. Information Systems Research, 11(4), 386-401. 
Lussier, R. N. (1995). A nonfinancial business success versus failure prediction model for young firms. Journal of Small Business Management, 33(1), 8.

March, J. (1978). Bounded Rationality, Ambiguity, and the Engineering of Choice. The Bell Journal of Economics, 9(2), 587-608. https://doi.org/10.2307/3003600.

Massey, A. P., \& Wallace, W. A. (1991). Focus groups as a knowledge elicitation technique: An exploratory study. IEEE Transactions on Knowledge and Data Engineering, 3(2), 193-200.

Möllers, T., Bansemir, B., Pretzl, M., \& Gassmann, O. (2017). Design and evaluation of a system dynamics based business model evaluation method. In International conference on design science research in information system and technology (S. 125-144). Springer.

Montazemi, A. R., Wang, F., Nainar, S. K., \& Bart, C. K. (1996). On the effectiveness of decisional guidance. Decision Support Systems, 18(2), 181-198.

Morana, S., Schacht, S., Scherp, A., \& Maedche, A. (2017). A review of the nature and effects of guidance design features. Decision Support Systems, 97(C), 31-42.

Morris, M., Schindehutte, M., \& Allen, J. (2005). The entrepreneur's business model: Toward a unified perspective. Journal of Business Research, 58(6), 726-735.

Nagar, Y., \& Malone, T. (2011). Making business predictions by combining human and machine intelligence in prediction markets. In ICIS 2011 proceedings, December 5, 2011, paper 20.

Nielsen, J. (1997). The use and misuse of focus groups. IEEE Software, 14(1), 94-95.

Nonaka, I., \& von Krogh, G. (2009). Perspective - Tacit knowledge and knowledge conversion: Controversy and advancement in organizational knowledge creation theory. Organization Science, 20(3), 635-652.

Ojala, A. (2016). Business models and opportunity creation: How IT entrepreneurs create and develop business models under uncertainty. Information Systems Journal, 26(5), 451-476.

Olson, D. L., Delen, D., \& Meng, Y. (2012). Comparative analysis of data mining methods for bankruptcy prediction. Decision Support Systems, 52(2), 464-473.

Osterwalder, A., \& Pigneur, Y. (2013). Designing business models and similar strategic objects: The contribution of IS. Journal of the Association for Information Systems, 14(5), 237-244.

Osterwalder, A., Pigneur, Y., \& Tucci, C. L. (2005). Clarifying business models: Origins, present. and future of the concept. Communications of the Association for Information Systems, 16(1), 1.

Ozer, M. (2009). The roles of product lead-users and product experts in new product evaluation. Research Policy, 38(8), 1340-1349.

Pagani, M. (2009). Roadmapping 3G mobile TV: Strategic thinking and scenario planning through repeated cross-impact handling. Technological Forecasting and Social Change, 76(3), 382-395.

Parikh, M., Fazlollahi, B., \& Verma, S. (2001). The effectiveness of decisional guidance: An empirical evaluation. Decision Sciences, 32(2), 303-332.

Pauwels, C., Clarysse, B., Wright, M., \& van Hove, J. (2016). Understanding a new generation incubation model: The accelerator. Technovation, 50, 13-24.

Peffers, K., Tuunanen, T., Rothenberger, M. A., \& Chatterjee, S. (2007). A design science research methodology for information systems research. Journal of Management Information Systems, 24(3), 45-77.

Riedl, C., Blohm, I., Leimeister, J. M., \& Krcmar, H. (2013). The effect of rating scales on decision quality and user attitudes in online innovation communities. International Journal of Electronic Commerce, 17(3), 7-36.

Sengupta, K., \& Abdel-Hamid, T. K. (1993). Alternative conceptions of feedback in dynamic decision environments: An experimental investigation. Management Science, 39(4), 411-428.

Shepherd, D. A., Williams, T. A., \& Patzelt, H. (2015). Thinking about entrepreneurial decision making: Review and research agenda. Journal of Management, 41(1), 11-46. 
Silver, M. S. (1991). Decisional guidance for computer-based decision support. MIS Quarterly, 15(1), 105-122.

Simon, H. A. (1955). A behavioral model of rational choice. The Quarterly Journal of Economics, 69(1), 99-118.

Song, M., Podoynitsyna, K., van der Bij, H., \& Im Halman, J. (2008). Success factors in new ventures: A meta-analysis. Journal of Product Innovation Management, 25(1), 7-27.

Sosna, M., Trevinyo-Rodríguez, R. N., \& Velamuri, S. R. (2010). Business model innovation through trial-and-error learning: The Naturhouse case. Long Range Planning, 43(2), 383-407.

Soukhoroukova, A., Spann, M., \& Skiera, B. (2012). Sourcing, filtering, and evaluating new product ideas: An empirical exploration of the performance of idea markets. Journal of Product Innovation Management, 29(1), 100-112.

Speier, C., \& Morris, M. G. (2003). The influence of query interface design on decision-making performance. MIS Quarterly, 27(3), 397-423.

Spiegel, O., Abbassi, P., Zylka, M. P., Schlagwein, D., Fischbach, K., \& Schoder, D. (2016). Business model development, founders' social capital and the success of early stage internet start-ups: A mixedmethod study. Information Systems Journal, 26(5), 421-449.

Strauss, A., \& Corbin, J. M. (1990). Basics of qualitative research: Grounded theory procedures and techniques. Sage Publications, Inc.

Teece, D. J. (2010). Business models, business strategy and innovation. Long Range Planning, 43(2-3), 172-194.

Tversky, A., \& Kahneman, D. (1974). Judgment under uncertainty: Heuristics and biases. Science, 185(4157), 1124-1131.

Veit, D., Clemons, E., Benlian, A., Buxmann, P., Hess, T., Spann, M., Kundisch, D., Leimeister, J. M., \& Loos, P. (2014). Business models. Business and Information Systems Engineering, 6(1), $45-53$.

Venable, J., Pries-Heje, J., \& Baskerville, R. (2016). FEDS: A framework for evaluation in design science research. European Journal of Information Systems, 25(1), 77-89.

Yuan, H., Lau, R. Y. K., \& Xu, W. (2016). The determinants of crowdfunding success: A semantic text analytics approach. Decision Support Systems, 91, 67-76.

Zhang, S. X., \& Cueto, J. (2017). The study of bias in entrepreneurship. Entrepreneurship Theory and Practice, 41(3), 419-454.

Zoric, J. (2011). Connecting business models with service platform designs: Exploiting potential of scenario modeling. Telematics and Informatics, 28(1), 40-54.

Zott, C., Amit, R., \& Massa, L. (2011). The business model: Recent developments and future research. Journal of Management, 37(4), 1019-1042. 

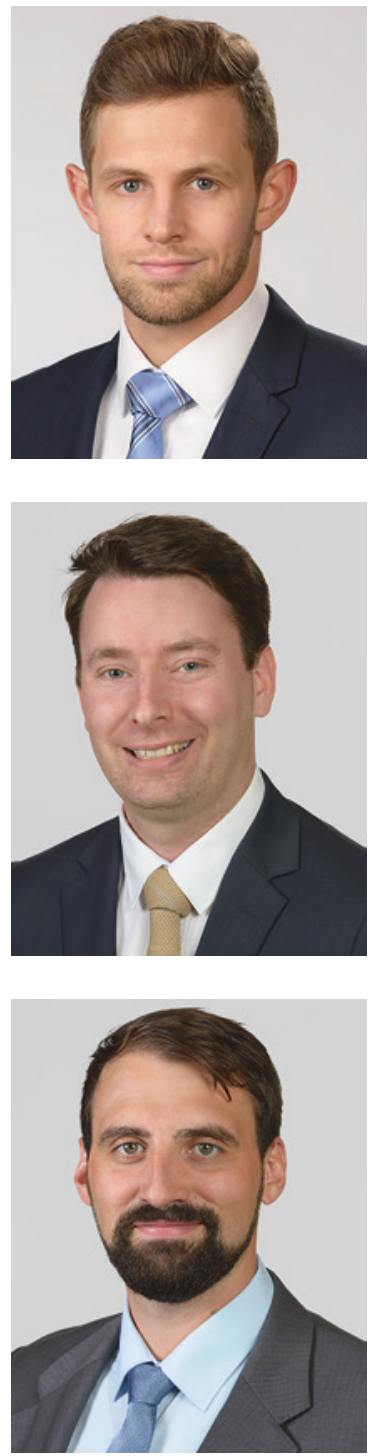

Dominik Dellermann studierte Betriebswirtschaftslehre (Abschluss als Master of Science) an der Universität Bamberg und der Universität Innsbruck mit dem Schwerpunkt Marktstrategien. Zuvor absolvierte er ein Studium International Business Studies (Bachelor of Arts) an der Universität Erlangen-Nürnberg und der Stockholm School of Economics. Nach Abschluss seines Studiums war er als wissenschaftlicher Mitarbeiter am Kompetenzzentrum für Geschäftsmodelle in der digitalen Welt an der Universität Bamberg tätig.

Nikolaus Lipusch studierte Betriebswirtschaftslehre an der Wirtschaftsuniversität Wien mit den Schwerpunkten Außenhandel und Entrepreneurship \& Innovation. Sein Masterstudium International Business Studies schloss er an der Friedrich-Alexander-Universität Erlangen-Nürnberg ab. Er absolvierte einen Auslandsstudienaufenthalt am Indian Institute of Management Bangalore. Seine Forschungsinteressen liegen im Bereich Open Innovation, insbesondere Crowdsourcing.

Dr. Philipp Ebel ist wissenschaftlicher Mitarbeiter und Leiter einer Forschungsgruppe am Institut für Wirtschaftsinformatik (IWI HSG) der Universität St. Gallen. Außerdem ist er Gastwissenschaftler am Fachbereich Wirtschaftsinformatik der Universität Kassel. Seine Forschung konzentriert sich auf agile Innovation, digitale Geschäftsmodelle und hybride Intelligenz. Er lehrt in verschiedenen Executive Education Programmen zu diesen Themen. Seit 2011 ist er als Berater, Trainer und Beiratsmitglied für verschiedene Unternehmen tätig. Seine Forschungsarbeiten wurden in zahlreichen renommierten internationalen Zeitschriften und Konferenzberichten veröffentlicht. 


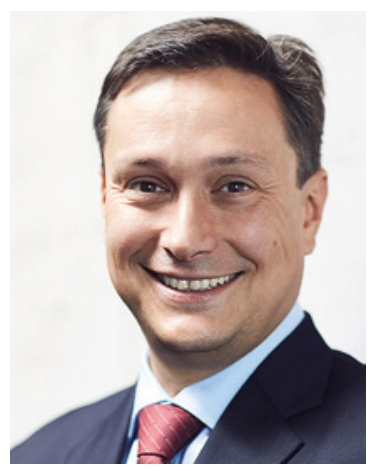

Prof. Dr. Jan Marco Leimeister ist Professor und Direktor am Institut für Informationsmanagement der Universität St. Gallen, Schweiz. Darüber hinaus ist er Professor und Direktor am Forschungszentrum für Informationssystem-Design (ITeG) an der Universität Kassel, Deutschland. Seine Forschung deckt die Bereiche Digital Business, Digital Transformation, Service Engineering und Service Management, Crowdsourcing, Digital Work, Collaboration Engineering und IT-Innovationsmanagement ab. Die Forschung und Lehre von Prof. Leimeister wurde mit verschiedenen Preisen ausgezeichnet, z. B. 2010 mit dem TUM Research Excellence Award und 2016 mit dem AIS Award for Innovation in Teaching. Die deutsche Wirtschaftszeitung „Handelsblatt" reiht seine Forschungsleistung seit 2009 kontinuierlich unter den Top $1 \%$ der Managementwissenschaftler in der deutschsprachigen Wissenschaftsgemeinschaft ein. 